\title{
1918/19. Markstein der deutschen Demokratie. Neuerscheinungen zum 100. Jahrestag der Revolution
}

\author{
Ulrich Wyrwa
}

Angenommen: 1. Juli 2020 / Online publiziert: 28. Januar 2021

(C) Der/die Autor(en) 2021

Zusammenfassung Während die deutsche Revolution von 1918/19 im Zuge des 50. Jahrestages Gegenstand heftiger Kontroversen war, ist sie bald darauf in Vergessenheit geraten. Der 100. Gedenktag führte zu einer Wiederentdeckung der Revolution. Sie gilt nicht mehr als verraten, steckengeblieben oder gar überflüssig, sie wird auch nicht mehr allein vom Ende der Weimarer Republik her als a priori zum Scheitern verurteilt abgetan, vielmehr als Aufbruch in die Demokratie gewürdigt.

Die Revolution ging jedoch nicht aus einer revolutionären Situation hervor, sondern, wie hier vorgestellte Neuerscheinungen zeigen [I.], aus dem Krieg. Die aus Anlass des 100. Jahrestages erschienenen neuen Gesamtdarstellungen [II.] weisen zumeist die alten Legenden von der verratenen Revolution zurück und betonen die Errungenschaften und demokratischen Potenziale, auch die Dynamik der revolutionären Situation, die Ängste der Akteure und die Gewaltgeschichte der Revolution. Regional- und stadtgeschichtliche Studien [III.] wiederum arbeiten die Unterschiede der revolutionären Entwicklungen und vor allem die Breite der Rätebewegungen heraus. Entscheidenden Einfluss auf die Entwicklung der Revolution hatte die Spaltung der Sozialdemokratie, Gegenstand weiterer Neuerscheinungen [IV.], aber nicht nur Arbeiter traten als Akteure hervor, sondern auch Intellektuelle [V.] und Künstler, wie hier besprochene Ausstellungskataloge zeigen [VI.].

Obgleich gelegentlich die alten Legenden von der verratenen Revolution bedient werden, erscheint die deutsche Revolution von 1918/19 zum 100. Jubiläum in einem neuen, zwar immer noch ambivalenten, zugleich aber positiveren Licht.

Das abschließende Resümee diskutiert die neuen Einsichten in die demokratischen Potenziale der Revolution, die Bedeutung der Rätebewegung, den Bolschewismus als die ,große Furcht“, das Umschlagen der friedlichen Revolution in Gewalt sowie das Aufkommen des extremen Antisemitismus.

U. Wyrwa (四)

Universität Potsdam, Potsdam, Deutschland

E-Mail:wyrwa@uni-potsdam.de 
Schlüsselwörter Die deutsche Revolution 1918/1919 · Rätebewegung ·

Sozialdemokratische Parteien · Konterrevolution · Demokratie · Weimarer Republik

\title{
1918/19. Milestone of German Democracy. New Publications on the 100th Anniversary of the Revolution
}

\begin{abstract}
On the 100th anniversary the old legends of the betrayed or failed revolution are rejected. General works show this revolution born of war as the dawn of democracy, regional studies examine the particularities. A sting is still the division of the socialist movement. Other publications investigate the revolutionary engagement of intellectuals and artists. The concluding remarks discuss the democratic potentials of the revolution, the significance of the council movement, Bolshevism as the 'great fear', the turn of the peaceful revolution to violence, and the emergence of extreme antisemitism.
\end{abstract}

Keywords German Revolution 1918/1919 · Council movement · Social Democracy parties · Counterrevolution · Democracy · Weimar Republic

Als Reinhard Rürup in Vorbereitung auf den 50. Jahrestag der Revolution von 1918/19 in dieser Zeitschrift einen Beitrag zum Verhältnis von Revolution und Rätebewegung veröffentlichte, konstatierte er eingangs, dass die Revolution von 1918/19 in der Wissenschaft „lange Zeit nur geringes Interesse gefunden“ hatte, in jüngster Zeit jedoch neue Aufmerksamkeit auf sich gezogen habe. ${ }^{1}$ Diese war, so betonte Rürup, vor allem auf das zuvor ,,vernachlässigte [...] Phänomen der revolutionären Arbeiter- und Soldatenräte" gerichtet. $^{2}$

Nach dem 50. Jahrestag ist im Kontext der politischen Debatten der 1970er Jahre eine große Zahl von Veröffentlichungen über die Rätebewegung erschienen, die vielfach im Tenor einer scharfen Verurteilung der sozialdemokratischen Politik in der schwierigen Gründungsphase der Republik geschrieben waren. Ihre Zuspitzung fand diese Deutung in dem 1969 erschienenen Essay von Sebastian Haffner mit der emphatischen, die These markant zusammenfassenden Überschrift „Die verratene Revolution“" 3

Das Revolutionsinteresse verfiel jedoch bald wieder, und so gab Alexander Gallus dem von ihm 2010 herausgegebenen Sammelband zur Revolution von 1918/19 den treffenden Titel „Die vergessene Revolution“. ${ }^{4}$ Schon 2016 aber konnte Volker Stal-

\footnotetext{
1 Rürup, Reinhard: Rätebewegung und Revolution in Deutschland 1918/19, in: Neue Politische Literatur 12 (1967), H. 3, S. 303-315, hier S. 303f.

2 Rürups Beitrag ist weniger Bericht über den Stand der Forschung als vielmehr ein ausführlicher Rezensionsessay zu der 1963 erschienenen Studie von Oertzen, Peter von: Betriebsräte in der Novemberrevolution. Eine politikwissenschaftliche Untersuchung über Ideengehalt und Struktur der betrieblichen und wirtschaftlichen Arbeiterräte in der deutschen Revolution 1918/19, Droste, Düsseldorf 1963.

3 Haffner, Sebastian: Die verratene Revolution - Deutschland 1918/19, Rowohlt, Hamburg 1969.

4 Gallus, Alexander (Hrsg.): Die vergessene Revolution von 1918/19, Vandenhoeck \& Ruprecht, Göttingen 2010 .
} 
mann von einer „Wiederentdeckung der Revolution“ sprechen. ${ }^{5}$ Zum 100. Jahrestag werden nun neue Fragen an die Revolution gestellt. Diese stehen im Zusammenhang mit einem frischen Blick auf die Weimarer Republik. „Bonn ist nicht Weimar“6, hieß es einst emphatisch in der alten Bundesrepublik Deutschland, die mit dieser negativen Zuschreibung sich selbst zu bestätigen und ihren Weg als Erfolgsgeschichte darzustellen suchte. Am Beginn des 21. Jahrhunderts ist dieses selbstgefällige Bild nachhaltig ins Wanken geraten. Die Krise der westlichen Demokratie hat Deutschland erreicht, und dieser Kontext eröffnete neue Einsichten in die erste deutsche Demokratie. Dabei standen zwei konträre Sichtweisen nebeneinander. Einerseits tauchte - anknüpfend an das Negativbild dieser Republik - in den öffentlichen Debatten die Frage auf, ob in Deutschland erneut „Weimarer Verhältnisse“ drohten. ${ }^{7}$ Andererseits wurden nun die positiven Momente der demokratischen Entwicklung der Republik und ihre Potenziale herausgestrichen. So trug die von der Historischen Kommission für Westfalen, dem Landesarchiv Nordrhein-Westfalen und der Gesellschaft für Rheinische Geschichtskunde am 9. November 2018 zum 100. Jahrestag der Revolution im Düsseldorfer Landtag veranstaltete Konferenz programmatisch den Titel: „Aufbruch in die Demokratie“. ${ }^{8}$ Der deutsche Bundespräsident FrankWalter Steinmeier eröffnete in diesem Sinne die Ausstellung des Deutschen Historischen Museums über die Weimarer Republik in Berlin mit der Warnung, dass diese ,nicht mehr nur von ihrem Ende her“ gedacht und erzählt werden dürfe, sondern ,ihre Verdienste, ihre Kreativität, ihre Aufbruchsenergien“ ebenfalls gewürdigt werden müssten. Die Republik sei „,keine Einbahnstraße in die Barbarei“ gewesen. ${ }^{9}$

Federführend in der Entwicklung dieses neuen Bildes der Weimarer Republik ist nicht zuletzt die von den Politikwissenschaftlern Michael Dreyer und Andreas Braune an der Universität Jena geleitete Forschungsstelle Weimarer Republik, die seit 2016 die Reihe „Weimarer Schriften zur Republik“ herausgibt. Eröffnet wurde diese mit dem Band ,Weimar als Herausforderung. Die Weimarer Republik und die Demokratie im 21. Jahrhundert". ${ }^{10}$ Die Republik hat, wie die Herausgeber schreiben, in den letzten Jahren an Aktualität gewonnen, und die Revolution von 1918/19 bezeichnen sie als ,erfolgreiche Revolution“. Ursula Büttner akzentuiert in ihrem Beitrag die beachtlichen Leistungen, ,die in den 14 Jahren der Weimarer Republik vollbracht wurden“ (S. 86). Nach den mittleren Jahren ,schien eine

\footnotetext{
5 Stalmann, Volker: Die Wiederentdeckung der Revolution von 1918/19. Forschungsstand und Forschungsperspektiven, in: Zeitschrift für Geschichtswissenschaft 64 (2016), H. 6, S. 521-541.

${ }^{6}$ So der immer wieder zitierte Titel des 1956 erschienenen Buches von dem Schweizer Journalisten Allemann, Fritz René: Bonn ist nicht Weimar, Kiepenheuer \& Witsch, Köln 1956.

7 So der Titel einer zwischen April und Juli 2017 erschienenen Artikelserie in der Frankfurter Allgemeinen Zeitung, die im Jahr darauf als Buch erschienen ist: Wirsching, Andreas/Kohler, Berthold/Wilhelm, Ulrich (Hrsg.): Weimarer Verhältnisse? Historische Lektionen für unsere Demokratie, Reclam, Stuttgart 2018.

${ }^{8}$ Siehe dazu jetzt den umfangreichen Tagungsband von Bischoff, Frank/Hitze, Guido/Reininghaus, Wilfried (Hrsg.): Aufbruch in die Demokratie. Die Revolution 1918/19 im Rheinland und in Westfalen (Veröffentlichungen der Historischen Kommission für Westfalen, N. F., Bd. 51), Aschendorff, Münster 2020.

9 Nach dem Bericht von Kilb, Andreas: Sie ist wieder da. Das Deutsche Historische Museum lobt die Verdienste der Weimarer Republik, in: Frankfurter Allgemeine Zeitung, 5. April 2019.

10 Dreyer, Michael/Braune, Andreas (Hrsg.): Weimar als Herausforderung. Die Weimarer Republik und die Demokratie im 21. Jahrhundert (Weimarer Schriften zur Republik, Bd. 1), Steiner, Stuttgart 2016.
} 
Konsolidierung auch der politischen Verhältnisse möglich zu sein“ (S. 91). Erst die Weltwirtschaftskrise und die „Politik des Kabinetts Brüning“ führten zur Zerstörung der Demokratie. Alexander Gallus knüpft in seinem Beitrag an den von ihm herausgegebenen Sammelband an und überprüft die These von der vergessenen Revolution angesichts der seit 2010 einsetzenden neuen Perspektiven auf Republik und Revolution. Er rekapituliert zunächst die Phasen der Revolutionsforschung und skizziert abschließend die „Perspektiven einer künftigen Revolutionsbetrachtung“ (S. 16). Nachdrücklich plädiert er dafür, sich den Wahrnehmungen und Erfahrungen der Zeitgenossen zuzuwenden und den Weg einer ,historisierenden Erfahrungsgeschichte“ (S. 9) einzuschlagen.

Mit diesem Band legten die Herausgeber eine fulminante Eröffnung der Reihe vor. Der im November 2018 erschienene und aus einer Tagung der Forschungsstelle hervorgegangene sechste Band der Reihe liefert grundlegende Beiträge über die Revolution vom Ereignis zum Erinnerungsort. ${ }^{11}$

\section{Vom Krieg zur Revolution}

Dass die Ursachen der Revolution von 1918 nicht in einer revolutionären Situation, sondern im Krieg lagen, hat der Doyen der historischen Forschung zum Ersten Weltkrieg, Gerd Krumeich, betont. ${ }^{12}$ In seinem im Herder Verlag erschienenen Band „Die unbewältigte Niederlage. Das Trauma des Ersten Weltkriegs und die Weimarer Republik“ arbeitet er heraus, wie die Weimarer Republik ,aus dem Krieg geboren“ ist und ,während ihrer gesamten Existenz ein Kind des Großen Krieges" blieb (S. 11). „Viel zu wenig“, hebt Krumeich hervor, ,wird gemeinhin beachtet, dass der entscheidende Umsturz des alten Systems - also die Revolution im engeren Sinne - von Soldaten ausgelöst wurde, die kein revolutionäres Programm hatten“ (S. 130). Am Anfang standen die Soldatenräte, erst dann bildeten sich Arbeiterräte. Das Trauma der nicht verstandenen und nicht angenommenen Niederlage und die ebenso wenig verarbeitete Frage der Kriegsschuld brachen dann in den „Schützengräben der Kriegserinnerung“ (S. 211) wieder hervor. Etwa 400.000 der sechs Millionen Soldaten, die nach 1918 zurückkehrten, so schätzt Krumeich, gaben sich einem ,soldatischen Nationalismus“ hin und waren bereit und willens, „den Krieg in die Zivilgesellschaft zu importieren“" (S. 230f.).

Zusammen mit Gerhard Hirschfeld und Irina Renz hat Krumeich den Band „1918. Die Deutschen zwischen Weltkrieg und Revolution“ herausgegeben. ${ }^{13}$ Aus einer großen Zahl von Ego-Dokumenten wie Briefen und Tagebuchaufzeichnungen von nichtrevolutionären Zeitgenossen haben sie „eine Art Kaleidoskop dieses Jahres“ zusam-

\footnotetext{
11 Braune, Andreas/Dreyer, Michael (Hrsg.): Zusammenbruch, Aufbruch, Abbruch? Die Novemberrevolution als Ereignis und Erinnerungsort (Weimarer Schriften zur Republik, Bd. 6), Steiner, Stuttgart 2019.

12 Krumeich, Gerd: Die unbewältigte Niederlage. Das Trauma des Ersten Weltkriegs und die Weimarer Republik, Herder, Freiburg i. Br. 2018.

13 Hirschfeld, Gerhard/Krumeich, Gerd/Renz, Irina (Hrsg.): 1918. Die Deutschen zwischen Weltkrieg und Revolution, Links, Berlin 2018.
} 
mengestellt und damit ihren Ansatz der Erfahrungs- und Wahrnehmungsgeschichte auf die Revolution ausgeweitet.

Das Erbe des Krieges nimmt der Journalist Andreas Platthaus, verantwortlicher Leiter der Bereiche Literatur und literarisches Leben der „Frankfurter Allgemeinen Zeitung“, in seinem Band „Der Krieg nach dem Krieg. Deutschland zwischen Revolution und Versailles 1918/19“ gleichfalls in den Blick. ${ }^{14}$ Er konzentriert sich dabei vor allem auf die „deutsche Verzweiflung“, die inneren Konflikte um den Friedensvertrag und den Kampf gegen die Republik. Die Revolution selbst blendet er nicht aus, sie steht aber nicht im Zentrum seiner Darstellung.

Zum Thema „Krieg nach dem Krieg“ hat der Fotohistoriker Anton Holzer einen großformatigen Band vorgelegt, komponiert aus zeitgenössischen Beobachtungen und Tagebucheinträgen sowie Fotografien, Bilddokumenten und Plakaten. ${ }^{15}$ Die Auswahl konzentriert sich vor allem auf Deutschland, doch richtet Holzer immer wieder den Blick zugleich auf Österreich und Ungarn, gelegentlich auf Russland. Zeitlich bezieht sich Holzer auf die Jahre von 1917 bis 1923, abschließend öffnet er einen Ausblick auf die späten 1920er Jahre. Es ging ihm bei der Zusammenstellung dieses Zeitpanoramas darum, den Erfahrungshorizont der Zeitgenossen einzufangen. Nach Holzers Kompositionskonzept sollten die autobiografischen Quellen ebenso für sich sprechen, wie die ausgewählten Fotografien. Verzichtet hat er - für einen Fotohistoriker erstaunlich - sowohl auf die Angaben der Fotografen als auch auf eine genaue Bildanalyse.

Einem anderen Aspekt des Zusammenhangs von Krieg und Revolution widmet sich der emeritierte Bremer Historiker Lothar Machtan. ${ }^{16}$ In seinem Buch „Kaisersturz“ geht er dem Ende der Hohenzollernmonarchie im November 1918 nach. Ohne die Arbeiter- und Soldatenräte zu übergehen wurde der „Kampf um die Vormacht“ aber nach Machtan ,zuallererst im deutschen Parlament geführt“ (S. 292). Die Straße als Schauplatz der Revolution taucht bei Machtan nur peripher auf. Die Dynamik der revolutionären Entwicklung, die gegensätzlichen politischen Konzepte und schließlich die Begründung der deutschen Republik bleiben ebenso unterbelichtet.

\section{Neue Gesamtdarstellungen der Revolution von 1918/19}

An neuen Gesamtdarstellungen sticht vor allem diejenige von Robert Gerwarth hervor. Er eröffnet diese mit der Bemerkung, dass die bisherigen negativen Urteile über die Revolution allein ,,vom retrospektiven Wissen um das Ende der Weimarer Republik“ gefällt wurden (S. 15). ${ }^{17}$ Eingangs betont er, dass die Revolution, der Sturz der

\footnotetext{
14 Platthaus, Andreas: Der Krieg nach dem Krieg. Deutschland zwischen Revolution und Versailles 1918/19, Rowohlt Berlin, Berlin 2018.

15 Holzer, Anton: Krieg nach dem Krieg. Revolution und Umbruch 1918/19, Theiss, Darmstadt 2017.

16 Machtan, Lothar: Kaisersturz. Vom Scheitern im Herzen der Macht 1918, Theiss/WBG, Darmstadt 2018.

17 Gerwarth, Robert: Die größte aller Revolutionen. November 1918 und der Aufbruch in eine neue Zeit, aus dem Engl. v. Alexander Weber, Siedler, München 2018.
} 
Hohenzollernmonarchie im November 1918, „nahezu gewaltfrei“ verlaufen ist. Sie brachte breite politische Partizipationsmöglichkeiten und weitreichende Freiheitsrechte und führte zu der ,wohl fortschrittlichsten Republik der Zeit“ (S. 10). Die Regierung Friedrich Eberts habe „das Kunststück“ fertiggebracht, „die revolutionäre Energie zu kanalisieren, im Angesicht einer nie dagewesenen Niederlage die öffentliche Ordnung aufrecht zu erhalten und Millionen von schwer bewaffneten Soldaten friedlich zu demobilisieren“" (S. 27).

Die ,überwältigende Mehrheit der Deutschen“, so Gerwarth, „,begrüßte die revolutionäre Umgestaltung Deutschlands“ (S. 13). Was die zeitgenössischen Beobachter dagegen beunruhigte, war die von ihnen sehr genau wahrgenommene Entwicklung in Russland. Sozialdemokraten waren über den ,grenzenlosen Terror“ der Bolschewiki - Gerwarth zitiert aus einer sozialdemokratischen Zeitung - gleichfalls erschüttert (S. 149). So musste aus der Sicht der Zeitgenossen die ,überaus reale Gefahr eines Bürgerkrieges gebannt werden“ (S. 187).

Die „Herkulesaufgabe“ der Regierung unter Ebert aber bestand darin, die sechs Millionen schwer bewaffneten und ,zutiefst verbitterten“ Soldaten zu demobilisieren (S. 193). Allein aus diesem Grund hatte Ebert sich entschlossen, eine Absprache mit der Obersten Heeresleitung unter General Wilhelm Groener zu treffen, die zumeist als „Pakt" bezeichnet, von Gerwarth aber als ,pragmatische Übereinkunft“ (S. 194) charakterisiert wird. Über die verfassungsmäßige und staatspolitische Zukunft Deutschlands sollte eine demokratisch gewählte verfassungsgebende Nationalversammlung entscheiden. Das Ergebnis der Wahlen zeigt nach Gerwarth, dass die Mehrheit der deutschen Bevölkerung ,einen demokratischen Neubeginn und nicht einen sozialrevolutionären Umsturz der Gesellschaft“" wollte (S. 221).

Doch die revolutionäre Entwicklung hatte ,politisch-soziale Phantasien [...] auf der extremen Linken“ befördert (S. 199). Schon im Winter 1918/19 war ,der schwelende Konflikt zwischen moderaten und radikalen Revolutionären in offene Gewalt“" umgeschlagen (S. 201). Gegen den wesentlich von Anhängern der Spartakus-Gruppe getragenen Januaraufstand ging Gustav Noske als Armee- und Marineminister mithilfe von militärischen Freiwilligenverbänden vor. So kam es zu der widersinnigen Konstellation, dass Noske zur Niederschlagung des Aufstandes genau jene Kräfte rekrutierte, die, wie Gerwarth schreibt, ,die Revolution von Anfang an mit glühendem Hass“ ablehnten und sich durch ihre brutale Gewalt auszeichneten (S. 209).

Dagegen setzte im Frühjahr 1919 ,eine zweite, radikalere Phase der Revolution ein“, die sich, wie Gerwarth unterstreicht, ,gegen die demokratisch legitimierte Weimarer Koalitionsregierung“ richtete (S. 235). Deutschland war in den Jahren von 1919 bis 1923 nach Gerwarth eine „streitbare Demokratie“. Das „brutale Vorgehen der Staatsmacht" gegen die linksextremen Aufstandsbewegungen hinterließ jedoch „eine Verbitterung“, ,die lange nachwirken sollte“ (S. 288). Mit der Niederschlagung dieser Aufstandsbewegungen brach die politische Gewalt des Rechtsterrorismus aus, die in politischen Morden kulminierte. Dieser Gefahr von rechts hat sich die Demokratie mit Erfolg entgegengestellt. „Am Ende des Jahres 1923“, mit diesem klaren Urteil schließt Gerwarth, „ließ sich schwer bestreiten, dass sich die Republik unter widrigsten Umständen behauptet hatte.“ So könne kaum von ,einer ,gescheiterten“ oder nur ,halbherzigen“ Revolution" gesprochen werden (S. 296). 
Der Historiker Wolfgang Niess, leitender Redakteur beim Südwestrundfunk, legte zum 100. Jahrestag ebenso eine - allerdings ohne Quellennachweise und Anmerkungen versehene - Gesamtdarstellung der deutschen Revolution vor. ${ }^{18}$,Ein Jahrhundert nach der größten Revolution in der deutschen Geschichte“, schreibt Niess, ,ist es höchste Zeit für einen Neuanfang“ ihrer Interpretation und für eine Widerlegung der Legenden über die Revolution, denen zufolge sie überflüssig gewesen oder verraten worden sei.

Nach Rückblicken auf die Sozialdemokratie im Kaiserreich und im Krieg bezeichnet Niess die Versuche einer Parlamentarisierung gegen Ende des Krieges als „großen Bluff“ (S. 90). Die Matrosenmeuterei markiert den Beginn der Revolution. Im Dezember 1918 setzten die gewalttätigen Konflikte zwischen der Regierung und linksextremen Kräften ein, die in den Januarkämpfen kulminierten. Bei deren Niederschlagung arbeitete die Regierung auf verhängnisvolle Weise mit dem Militär zusammen. Es sollte sich sehr bald rächen, dass Ebert die Oberste Heeresleitung ,als Partner der Regierung akzeptiert und nicht von Anfang an auf dem Primat der Politik“ bestanden hat (S. 198). Es folgte die Gegenrevolution mit dem KappPutsch; als dieser gescheitert war, konnte die, so die emphatische Kapitelüberschrift, „demokratischste Demokratie der Welt“ aufgebaut werden.

Der Historiker und Journalist Joachim Käppner hat zum 100. Jahrestag der Revolution gleichfalls eine neue Gesamtdarstellung vorgelegt, die sich ebenfalls schon im Titel von den älteren Sichtweisen abgrenzt. Es handele sich bei den Ereignissen von 1918 um einen „Aufstand für die Freiheit“, um eine „Revolution der Besonnenen“. ${ }^{19}$

„Selten hat eine Revolution leichter gesiegt“, so Käppner, aber „,selten hat eine Revolution auch solch katastrophale Startbedingungen gehabt: eine vom Krieg zutiefst erschütterte und gespaltene Gesellschaft, voller Not und Hunger im Schatten eines Krieges, der verloren und nicht vorüber ist“" (S. 206). Die SPD habe es jedoch versäumt, „der Freiheit Waffen zu geben“. „Schuld daran ist eine obsessive Angst vor dem Bolschewismus." Käppner erinnert daran, dass die Arbeiter- und Soldatenräte „für eine parlamentarische Demokratie und gegen eine Diktatur der Räte“ votierten (S. 454). Obwohl es sich an anderer Stelle abweichend liest, spricht Käppner in seinem Resümee vom Scheitern der Revolution. Zur „Symbolfigur des Scheiterns“ erklärt er Gustav Noske. Als den „Sündenfall“ der Revolution macht Käppner schließlich den Moment aus, ,,als die Regierung [...] ein Heer aus Freiwilligen aufstellen lässt, die sich zum größten Teil als Todfeinde der Republik erweisen“ (S. 460).

Emphatisch fällt das Urteil der Historiker und Journalisten Sven Felix Kellerhoff und Lars-Broder Keil aus, die schon im Titel das „Lob der Revolution“ ausbringen. ${ }^{20}$ Ihre Erzählung setzt mit dem Eingeständnis der Niederlage durch die Oberste Heeresleitung im September 1918 ein und behandelt dann den Waffenstillstand, die

\footnotetext{
18 Niess, Wolfgang: Die Revolution von 1918/19. Der wahre Beginn unserer Demokratie, Europa, München/Wien 2017.

19 Käppner, Joachim: 1918. Aufstand für die Freiheit. Die Revolution der Besonnenen, Piper, München 2017.

20 Keil, Lars-Broder/Kellerhoff, Sven Felix: Lob der Revolution. Die Geburt der deutschen Demokratie, Theiss/WBG, Darmstadt 2018.
} 
Regierungsumbildung in Berlin sowie die Furcht vieler Sozialdemokraten vor dem Bolschewismus und vor einem Bürgerkrieg. Der Einfluss linksextremer Sozialisten und der Spartakusgruppe war aber gering. Auf dem Kongress der Arbeiter- und Soldatenräte zeigte sich, dass diese kaum Rückhalt in der revolutionären Bewegung hatten. Die Rückkehr der Frontsoldaten und die Demobilisierung des Heeres stellte die Regierung vor Herausforderungen, die sie glaubte, nur mithilfe der neuen Obersten Heeresleitung lösen zu können. Die sich in den deutschen Städten bildenden Rätestrukturen nahmen an der Verwaltung teil, über deren Zukunft allerdings Unklarheit herrschte. Die ersten Wochen des Umsturzes waren weitgehend gewaltfrei, doch in Berlin spitzte sich die Lage Anfang Dezember zu. Mit dem aussagekräftigen Zitat von Egon Erwin Kisch: „Plötzlich ist der Krieg auf Deutschlands Boden“ eröffnen Kellerhoff und Keil das Kapitel über den Januaraufstand, mit dem ,im revolutionären Deutschland der latente Bürgerkrieg" begonnen habe (S. 124). Gleichwohl konnten die Wahlen zur Nationalversammlung durchgeführt und eine Verfassung verabschiedet werden. Auf der Linken hingegen verbreitete sich, wie Kellerhoff und Keil ausführen, die vor allem vom USPD-Politiker Emil Barth entwickelte Legende vom Verrat der SPD.

Im markanten Gegensatz zu den bisher vorgestellten Gesamtdarstellungen vertritt Bernd Langer in seinem Band „Die Flamme der Revolution“ noch einmal genau diese Legende. ${ }^{21}$ Geschrieben ist das Buch mit unverhohlener Empathie für die linksextremen Bewegungen und mit augenfälligen Vorurteilen gegenüber der SPD. Dass sich die SPD auf der ersten Versammlung der Arbeiter- und Soldatenräte am 10. November durchsetzte und eine paritätisch aus Mitgliedern von MSPD und USPD zusammengesetzte Regierung bildete, bezeichnet Langer als „Fiasko“ (S. 156). Im Beschluss des Reichsrätekongresses vom Dezember 1918, im Januar Wahlen zur verfassungsgebenden Nationalversammlung abzuhalten, sieht Langer eine „eklatante Niederlage“ der Linken (S. 195). Nach den Weihnachtskämpfen 1918 hätten ,Aktivisten der Straße“ in ,Eigeninitiative“ den „Aufstand auf die Tagesordnung gesetzt“ (S. 241). Die Besetzung der Redaktion des ,Vorwärts“ und weiterer Verlagshäuser durch die Aufständischen wurde dann vom Militär und von Freikorps niedergeschlagen. „Für die revolutionäre Bewegung“, so Langers Fazit, war „eine schwere Niederlage zu verkraften“ (S. 297). Die nur eine Woche darauf stattfindenden Wahlen zur Nationalversammlung dokumentieren, wie Langer zugestehen muss, „dass die radikalen Linken keine Mehrheit in der Bevölkerung" besaßen (S. 323).

Dennoch setzte in anderen Städten die linksextreme Bewegung ihre Aktionen fort. Sie wurden jedoch, wie Langer erbittert schreibt, unter dem Oberbefehl von Gustav Noske mit militärischer Gewalt und mithilfe von Freikorps niedergeschlagen. Trotz aller Empathie mit den linksextremen Bewegungen übt Langer Kritik an der ultralinken Programmatik der KPD; immerhin hatte sie, wie er anerkennend betont, „die Unterstützung der Bolschewiki“ (S. 426). „Zu Beginn des Jahres 1920 ist die Lage im Deutschen Reich“ - mit diesem Satz beendet Langer seinen kurzen, „Der Kampf geht weiter“ überschriebenen Abschnitt - ,weiterhin instabil“ (S. 431).

Wider Willen macht diese Darstellung der dramatischen Gründungsphase der Weimarer Republik die Furcht der Regierung Friedrich Ebert und Philipp Scheide-

${ }^{21}$ Langer, Bernd: Die Flamme der Revolution. Deutschland 1918/19, Unrast, Münster 2018. 
mann vor einem ,russischen Weg“ verständlich. Wenn in der westdeutschen Geschichtswissenschaft seit den 1970er Jahren vielfach geschrieben wurde, dass in Deutschland keine zweite Revolution nach bolschewistischem Modell gedroht habe, belegen die von Langer so ausführlich dokumentierten Aktivitäten der deutschen linksextremen Bewegungen, wieso aufseiten der Sozialdemokratie ebenso wie im liberalen Lager tiefe Ängste vor einer russischen Entwicklung verbreitet waren.

Einen ähnlichen Ansatz wie Langer verfolgt der Sozialwissenschaftler und Regisseur Klaus Gietinger, der zuvor eine Studie über den Mord an Rosa Luxemburg, sowie eine Biografie über den Freikorpsführer Waldemar Pabst vorgelegt hat. ${ }^{22}$ Seine Revolutionsgeschichte ist eine erzürnte Anklageschrift gegen die Sozialdemokratie. ${ }^{23}$ Die entscheidenden Akteure der Revolution waren, nach Gietinger, die revolutionären Obleute. Trotzdem gelang es der SPD, die Arbeiter und Soldaten für sich zu gewinnen. Diese vergaßen jedoch, so Gietinger, ,dass sich die SPD-Führung mit ihrer Kriegsunterstützung und Ablehnung jeder Revolution [...] korrumpiert hatte“ (S. 63). So konnte sie auf dem Kongress der Arbeiter- und Soldatenräte eine paritätische Regierung aus SPD und USPD durchsetzen. Gleichzeitig sei Friedrich Ebert zielstrebig darangegangen, ein Bündnis mit der Obersten Heeresleitung aufzubauen. Die unabhängigen Sozialisten seien mit dem Austritt aus der Regierung, in eine Falle getappt“, die ihr die SPD gestellt habe. An ihre Stelle trat nun Gustav Noske in die Regierung. Die von ihm aufgebauten Freikorps waren „Truppen neuen Typs“, ,autoritär geführte Stoßtrupps mit hoher Zerstörungskraft“, deren „,Vernichtungswille [...] nun auf den inneren Feind konzentriert und durch den Mythos von der Abwehr des Bolschewismus zusätzlich aufgeladen“"wurde (S. 105). Dass die SPD in der Wahl zur verfassungsgebenden Nationalversammlung 37,9\% der Stimmen erzielte, kommentiert Gietinger mit der knappen Bemerkung: „Noch glaubten viele an die SPD. [...] Noch begriffen viele der SPD-Anhänger nicht, was ihre Oberen wollten“ (S. 137). Die in Weimar verabschiedete Verfassung wertet Gietinger als ein ,autoritäres, teils nach Bismarck'schem Vorbild gebautes Grundgesetz“ ab (S. 193).

Während die Gewaltgeschichte der Revolution in Gietingers Darstellung mit scharfen Anklagen gegen die Sozialdemokratie verbunden ist, hat der irische Historiker Mark Jones diese Geschichte mit - so Gietinger - ,,irischer Klarheit“ (S. 164) analysiert. ${ }^{24}$ Das Verhängnis dieser Gewaltgeschichte lag für Jones im Gegensatz zu Gietinger jedoch daran, dass die ,,politischen Führer“ der Revolution , und große Teile der damaligen deutschen Gesellschaft“ ,,aus Angst, Opfer revolutionärer Gewalt zu werden, am Ende die Anwendung [...] kriegsmäßiger Gewalt [...] gegen den (wirklichen oder vermeintlichen) inneren Feind“ billigten (S. 14). Während Gietinger die Sozialdemokratie anklagt, geht es Jones darum, ,ein Verständnis dafür zu gewinnen, wie und weshalb die politischen Akteure zu den Entscheidungen kamen“. Ausschlaggebend war, dass die deutsche Sozialdemokratie sehr genau wusste, ,,was

\footnotetext{
22 Gietinger, Klaus: Eine Leiche im Landwehrkanal. Die Ermordung Rosa Luxemburgs, Nautilus, Hamburg 2009; ders.: Der Konterrevolutionär. Waldemar Pabst - eine deutsche Karriere, Nautilus, Hamburg 2009.

23 Ders.: November 1918. Der verpasste Frühling des 20. Jahrhunderts, Nautilus, Hamburg 2018.

24 Jones, Mark: Am Anfang war Gewalt. Die deutsche Revolution 1918/19 und der Beginn der Weimarer Republik, aus dem Engl. v. Karl Heinz Siber, Propyläen, Berlin 2017 (engl. 2016).
} 
der Bolschewismus war: eine Spielart des Terrorismus“ (S. 21). Im Unterschied zu Russland zeichnete sich der deutsche November 1918 trotz vereinzelter Vorfälle von Gewalt durch ein sehr niedriges Gewaltniveau aus. Nach dem November nahm jedoch die ,große Furcht“ in dem „Schreckgespenst“ des Spartakismus Gestalt an. In der Revolution fand Karl Liebknecht mit seiner Forderung, dem russischen Vorbild zu folgen, zwar keinerlei Rückhalt, er verstärkte aber in der Öffentlichkeit und in der Sozialdemokratie die Angst vor dem Bolschewismus. Diese ,durch Gerüchte und autosuggestive Überzeugungen angeheizten Ängste zeitigten“, so Jones, ,politische Konsequenzen“ (S. 92). Nach den Erfolgen der Linksextremen in den Weihnachtsunruhen 1918 waren die Sozialdemokraten bereit, „die rote Linie zu überschreiten, die sie bis jetzt davon abgehalten hatte, den Militärs den Einsatz kriegerischer Mittel gegen die Revolution zu gestatten“ (S. 147). Dass linke, bewaffnete Kräfte in der „Euphorie des Augenblicks“ (S. 159) zum Kampf gegen die Regierung EbertScheidemann aufriefen, führte zu einer Radikalisierung der politischen Rhetorik und einer Steigerung der Gewaltbereitschaft. Rosa Luxemburg hatte an dieser Eskalation, wie Jones unterstreicht, ihrerseits „,mit ihrer kompromisslosen Aufforderung, Verhandlungen abzulehnen und stattdessen weiter auf die Option der revolutionären Gewalt zu setzen“" erheblichen Anteil (S. 173). Was die Sozialdemokraten dazu veranlasste, mit militärischer Gewalt gegen den Aufstand vorzugehen, war nicht zuletzt der Vorsatz, ,dass es den Sozialdemokraten nicht so ergehen würde wie Lenins sozialistischen Rivalen“ (S. 178). Jones erklärt die unerbittliche Härte, mit der sozialdemokratische Politiker den Einsatz von Gewalt legitimierten, aus der kognitiven Dissonanz zwischen den Nachrichten aus Russland und den damit ebenso bedrohlich erscheinenden Gerüchten über die Beteiligung von russischen Bolschewisten an dem Aufstand.

Die Streikbewegung vom März 1919 führte zu einer „Spirale der Gewalt“, in deren Zug Noske einen Schießbefehl erließ. Damit hatte, so Jones, die „Logik der Gewalt [...] über das Recht und über die Bürgerrechte triumphiert" (S. 253). Und doch eröffnet Jones seinen Epilog mit der Feststellung, dass die Republik ,,schon ein knappes Jahr nach der Revolution [...] auf recht festem Boden“ stand (S. 331). Die Revolution schuf gar „eines der stabilsten politischen Ordnungssysteme“ in Mitteleuropa (S. 331). Die ,,von Regierungstruppen und Freikorps begangenen Gewaltexzesse“ in der Gründungsphase der Republik (S. 336) waren zudem, wie Jones in seinem Schlusswort hervorhebt, kein Sonderfall in Europa.

Der damit angedeutete europäische Vergleich steht im Fokus von Kersten Knipps Darstellung, die 1918 dezidiert als ein, so der Untertitel, ,europäisches Schicksalsjahr" präsentiert. ${ }^{25}$ Knipp geht von der Hinterlassenschaft des Krieges aus und schildert, wie im Krieg auf diplomatischen Wegen die Weichen für eine neue europäische Ordnung geplant und vor allem in Ostmitteleuropa die Gründung neuer Staaten vorbereitet wurde. Nicht zuletzt mit der vom US-amerikanischen Präsidenten Woodrow Wilson auf eine ,robuste Demokratie“ zielenden Initiative kam der Wunsch nach einem dauerhaften Frieden in Europa auf. In Paris aber musste Wilson erfahren, dass seine Vorstellungen ,mit den politischen Realitäten [...] in eine kaum lösbare Spannung gerieten“ (S. 180). Diese zeichnet Knipp für den europäischen

${ }^{25}$ Knipp, Kersten: Im Taumel. 1918 - ein europäisches Schicksalsjahr, Theiss, Darmstadt 2018. 
Raum nach, wobei er neben den innerdeutschen Konfliktlinien vor allem die vielfältigen Gegensätze, die sich im ostmitteleuropäischen Raum durch das Ende der Habsburgermonarchie ergaben, rekonstruiert. Welche Probleme bei der Schaffung einer neuen Ordnung aufbrachen, zeigt Knipp am Beispiel Österreichs, der „Zornigen Republik“. Die „Stunde der Autokraten“ hatte aber nicht nur dort, sondern auch in der Türkei und in Griechenland geschlagen.

Über die von Knipp ausgemessenen europäischen Dimensionen des Revolutionsjahres 1918 hinaus reicht der von Stefan Rinke und Michael Wildt herausgegebene Sammelband bis ins Globale. Er setzt mit der Russischen Revolution von 1917 ein und fragt nach dem Verhältnis von Revolution und Konterrevolution in globalgeschichtlicher Perspektive. ${ }^{26}$ Nach dem einleitenden historischen Rückblick der Herausgeber beleuchtet Jörn Leonhard die globalen Dimensionen des revolutionären Geschehens und die Erwartungen, die dadurch ausgelöst wurden. Zur Russischen Revolution, Ausgangspunkt des Bandes, arbeitet Dietrich Beyrau die gnadenlose Gewaltpolitik heraus, mit der die Bolschewiki ihre Diktatur ausbauten. Der erfolgreiche staatliche Terror wurde, wie Jan Claas Behrends zeigt, zum Vorbild für weitere diktatorische Regime in der Welt. Der Gewaltpolitik der europäischen konterrevolutionären Bewegungen geht Robert Gerwarth bis zum Ende der 1930er Jahre nach. Darüber hinaus präsentiert der Band die revolutionären und konterrevolutionären Entwicklungen in China und Lateinamerika.

\section{Regionalgeschichtliche Studien}

Schon die Ausbreitung der Revolution von Kiel über Lübeck, Bremen, Rostock, Hannover, Braunschweig, Bielefeld, Essen und Düsseldorf bis nach Stuttgart, München und Berlin, um nur einige Städte zu nennen, macht deutlich, welche Bedeutung die räumlichen Dimensionen für die Entwicklung des revolutionären Geschehens hatte. Ein Blick auf die innerstädtischen Bezüge und die Abläufe der revolutionären Entwicklungen vor Ort wiederum zeigen die bisher nur unzureichend ausgeleuchteten Räume des revolutionären Geschehens.

Den Räumen der Revolution hat sich Julian Aulke unter kulturwissenschaftlicher Perspektive zugewandt. ${ }^{27}$ Ihm geht es vor allem darum, die Konzentration auf die politischen und ideologischen Konflikte der Revolution zu überwinden und mit kulturalistischen Methoden zu erfassen, wie im Zuge der revolutionären Entwicklung Räume kulturell besetzt wurden und die Akteure diese durch ihr Handeln symbolisch aufluden. Aulke beschränkt sich dabei auf großstädtische Zentren der Revolution; im Mittelpunkt stehen Berlin, die Städte Westfalens und des Rheinlandes sowie München. Der Gang seiner Untersuchung über die räumlichen Dimensionen des Revolutionsgeschehens geht von den Straßen, Stadtvierteln und Wirtshäusern als Arenen politischer Kämpfe über zu den divergierenden Praktiken, mithilfe derer

\footnotetext{
26 Rinke, Stefan/Wildt, Michael (Hrsg.): Revolutions and Counter-Revolutions. 1917 and its Aftermath from a Global Perspective, Campus, Frankfurt a. M./New York 2017.

27 Aulke, Julian: Räume der Revolution. Kulturelle Verräumlichung in Politisierungsprozessen während der Revolution 1918-1920 (Studien zur Geschichte des Alltags, Bd. 31), Steiner, Stuttgart 2015.
} 
Ordnung und Sicherheit in den Stadträumen geschaffen wurde. Schließlich nimmt er die kommunikative und symbolische Besetzung der Räume in den Blick.

Von dieser kulturwissenschaftlichen Studie über die „Räume der Revolution“ sind konkrete lokalgeschichtliche Untersuchungen über die revolutionäre Entwicklung in Städten und Regionen zu unterscheiden. Der Kieler Historiker und Publizist Martin Rackwitz hat eine Geschichte der Revolution in Kiel, dem Ursprungsort der deutschen Revolution, vorgelegt, in der er den Ursachen und dem Verlauf des Kieler Matrosen- und Arbeiteraufstandes nachgeht. ${ }^{28}$ Nach der akkuraten Rekonstruktion der Ereignisse vom 1. bis zum 10. November 1918 geht Rackwitz zentralen Fragen der revolutionären Entwicklung nach, so der Rolle von Gustav Noske im Kieler Soldatenrat, dem von der MSPD dominierten Arbeiterrat, dem Kieler Spartakistenaufstand und schließlich dem erfolgreichen Widerstand der Sozialdemokratie und der Gewerkschaften gegen den Kapp-Lüttwitz-Putsch. Die Kieler Bevölkerung aber blieb, wie Rackwitz festhält, gespalten.

Das Kieler Stadt- und Schifffahrtsmuseum hat zum 100. Jahrestag eine umfängliche Ausstellung gezeigt, die eine „Neuverortung des Matrosenaufstandes“ bot. Sie folgt nicht mehr den überkommenen Narrativen, sondern führt den Aufstand positiv in die Demokratisierungsgeschichte Deutschlands ein. Der von Sonja Kinzler und Doris Tillman herausgegebene Begleitband zur Ausstellung rekonstruiert minutiös die politischen Ereignisse in der Stadt, fragt nach der Rolle Kieler Matrosen in der raschen Ausbreitung der Revolution und zeigt das Engagement von Kieler Frauen für das Frauenwahlrecht sowie den Einfluss der Russischen Revolution auf Kiel. ${ }^{29}$ Schließlich wird die Formierung paramilitärischer Verbände und die Entwicklung konterrevolutionärer Gewalt in Kiel thematisiert. Beigegeben sind dem Band über 200 Fotografien, Plakate, Flugblätter, Zeichnungen und Gemälde, die fundiert erläutert und in den historischen Kontext gestellt werden.

Wie schon Rackwitz herausgearbeitet hat, verbreitete sich die revolutionäre Bewegung durch die aufständischen Matrosen sehr rasch in den Städten Norddeutschlands. Der an der Freien Universität Berlin lehrende Politikwissenschaftler Detlef Lehnert hat daher einen Band mit Beiträgen über die Revolution in sechs norddeutschen Hansestädten vorgelegt. ${ }^{30}$ Gemeinsame Quellengrundlage aller sechs Beiträge sind die lokalen Tageszeitungen, deren ,lückenlose Durchsicht“ (S. 12) nicht nur die Dynamik der revolutionären Entwicklung, sondern auch die politischen Meinungskämpfe in den Städten deutlich werden lasse. Die starke Politisierung der Bevölkerung zeigte sich etwa, wie Lehnert ausführt, in der Verdreifachung der Mitgliederzahlen der sozialdemokratischen Gewerkschaften. Der ,Übergang vom Kaiserreich zur Weimarer Republik“ war, so das pointierte Urteil von Lehnert, „eine (mehrheits-)sozialdemokratische Angelegenheit“" (S. 19).

\footnotetext{
28 Rackwitz, Martin: Kiel 1918. Revolution - Aufbruch zu Demokratie und Republik, Wachholtz, Kiel 2018.

29 Kinzler, Sonja/Tillmann, Doris: Die Stunde der Matrosen. Kiel und die deutsche Revolution 1918, Theiss/WBG, Darmstadt 2018.

30 Lehnert, Detlef (Hrsg.): Revolution 1918/19 in Norddeutschland (Historische Demokratieforschung, Bd. 13), Metropol, Berlin 2018.
} 
In diesem Sinne zeigt Karl Heinrich Pohl, dass es der SPD in Kiel gelang, eine „,von der Mehrheit der Bevölkerung getragene parlamentarische Demokratie“ durchzusetzen. Pohl bezeichnet diese ,unaufgeregte Strategie“ „als beispielhaft für den Weg Deutschlands“ in die Demokratie (S. 98). Die Situation in Hamburg war, wie Volker Stalmann in seinem Beitrag herausarbeitet, komplizierter. Trotz der ersten Erfolge der sozialdemokratischen Bewegung im Übergang zur parlamentarischen Republik standen ihr der alte Senat der Stadt Hamburg sowie linksradikale Akteure gegenüber, die beide nach Stalmann einen ,dezidiert antiparlamentarischen Standpunkt bezogen“ und eine „Putschtaktik“ verfolgten (S. 173).

Hans Rudolf Wahl rekonstruiert „Novemberrevolution, Räterepublik und Demokratiegründung in Bremen“. Was Bremen in dieser Phase auszeichnete, war die starke Präsenz der linksradikalen Bewegung. Obwohl die SPD bei den Wahlen zum Arbeiter- und Soldatenrat die Mehrheit der Stimmen erhielt, erzeugte vor allem die KPD, so Wahl, „ein militant aufgeladenes politisches Klima“ (S. 215). Mit der Rückkehr von Offizieren und Frontsoldaten, die vor dem Krieg in Bremen stationiert waren, trat ein weiterer entscheidender Akteur auf. Wie Wahl in seinem Fazit festhält, war die Bremer Räterepublik ,der schnell und blutig gescheiterte Versuch der Bremer KPD, ihren politischen Monopolanspruch nach dem Vorbild Lenins mit Gewalt durchzusetzen“ (S. 239).

Der Herausgeber Detlef Lehnert rekapituliert die Entwicklung der Revolution in Lübeck, „,wo der Umsturz der Verhältnisse“, wie er aus einem Artikel im Lübecker Generalanzeiger zitiert, ,unter verhältnismäßig geringen Erschütterungen vor sich gegangen“ ist (S. 252). Die Revolution in Rostock verlief, wie Axel Weipert nachzeichnet, ebenfalls „,in geordneten Bahnen“, so die aus der lokalen SPD-Zeitung übernommene Formulierung. Den Abschluss des Bandes bildet der Beitrag von Bernd Rother über die Entwicklung in der Hansestadt Lüneburg, wo die Politik der Sozialdemokraten zunächst ebenfalls auf eine Reform zielte, dann aber aufgrund des Widerstandes der alten Mächte eine revolutionäre Politik einschlug. Dass die SPD keine offene Auseinandersetzung mit der kaiserlichen Verwaltung und Militärführung suchte, wurde von den Wählern Lüneburgs honoriert. So verstärkte die Politik der Lüneburger Sozialdemokratie „das Vertrauen in die Partei“ (S. 382).

Die Entwicklung der Revolution in den Ostseestädten Stralsund, Greifswald und Stettin werden in einem von Thomas Stamm-Kuhlmann herausgegebenen und in der Schriftenreihe der Historischen Kommission für Pommern erschienenen Band vorgestellt. ${ }^{31}$ Diese Lokalstudien zeigen zum einen die Breite der Rätebewegung. So weist Jenny Linck in ihrem Beitrag über Greifswald auf eine Statistik des Oberpräsidenten der Provinz Pommern hin, der zufolge sich in 231 Städten und Gemeinden Arbeiter- und Soldatenräte sowie Bauernräte bildeten (S. 185). Darüber hinaus machen sie die politische Dynamik deutlich. In allen drei Städten ging die Initiative von den Räten aus, und diese arbeiteten bereitwillig mit den kommunalen Verwaltungen zusammen. So setzten sich in Greifswald die Vertreter der Arbeiterschaft mit dem vom Bürgermeister gegründeten Volksausschuss zusammen. Schließlich habe sich

\footnotetext{
31 Stamm-Kuhlmann, Thomas (Hrsg.): November 1918. Revolution an der Ostsee und im Reich (Veröffentlichungen der Historischen Kommission für Pommern. Reihe V: Forschungen zur Pommerschen Geschichte, Bd. 53), Böhlau, Köln u. a. 2020.
} 
der Arbeiter- und Soldatenrat in Greifswald nicht zuletzt deshalb gebildet, um, Linck zitiert eine Aussage des Vorsitzenden, ,den Bolschewismus zu verhindern“ (S. 182). Christoph Freiherr von Houwald macht in seinem Beitrag über Stralsund auf einen „Huldigungsumzug“ aufmerkam, mit dem ,eindrucksvoll der Schulterschluss zwischen den Protagonisten des [...] Arbeiter- und Soldatenrats und den städtischen Behörden inszeniert" wurde (S. 147). Wie in Greifswald und Stralsund herrschte unter den Arbeitern und Soldaten der Provinzhauptstadt Stettin, wie Gunter Dehnert zeigt, ebenfalls die Sorge vor, ,die revolutionäre Dynamik könne außer Kontrolle geraten“ (S. 196), wobei Dehnert zugleich auf die „Diskrepanz zwischen der revolutionären Rhetorik und wenig revolutionären Praxis“ der Arbeiterbewegung in Stettin hinweist (S. 198). So war der Arbeiter und Soldatenrat bereit, auf die ,Vertreter der alten Ordnung“" zuzugehen, diese aber versahen ihren Dienst, wie Dehnert abschließend festhält, offensichtlich nur „unter gewaltigem innerem Protest“ (S. 208).

Wenn Hans Rudolf Wahl in dem Band „Revolution 1918/19 in Norddeutschland“ eine überaus kritische Sicht auf den tendenziell undemokratischen Charakter der Bremer Räterepublik und die autoritären Züge der Politik der Bremer Linksradikalen formuliert hatte, so gibt der 1938 geborene und 2018 verstorbene Bremer Historiker Peter Kuckuk eine von großer Empathie für die Bremer Linksradikalen getragene Darstellung der Ereignisse. Schon 1969 hatte er einen Band mit Quellen über die Revolution und Räterepublik in Bremen herausgegeben. ${ }^{32} 1986$ legte er eine umfangreiche Studie über „Bremen in der Deutschen Revolution“ vor. 2017 ist anlässlich der Einhundertjahrfeier eine überarbeitete und um weitere Kapitel von Kuckuk sowie Ulrich Schröder erweiterte Ausgabe erschienen. ${ }^{33}$ Der Schwerpunkt der Darstellung liegt auf den linksradikalen und kommunistischen Akteuren, Sozialdemokraten beider Richtungen treten eher in den Hintergrund. Polemisch richtet sich Kuckuk gegen den, wie er sich ausdrückt, „Fetischcharakter des praktizierten Parlamentarismus“ (S. 13). Er schildert detailliert die Entwicklung in Bremen von der „Militärrevolte“ am 6. November 1918, der Bildung von Arbeiterräten und der Doppelherrschaft der alten und neuen Gewalten, der eine kurze Phase der Zusammenarbeit folgte. Mit dem Austritt der Sozialdemokraten aus den Räten Ende Dezember setzte eine neue Phase in der Bremer Revolutionsgeschichte ein, die zur Ausrufung der Räterepublik durch USPD und KPD führte, nach Kuckuk der ,Übergang zur Diktatur des Proletariats“ (S. 321). Zwar kritisiert Kuckuk die ,destruktiven Aktionen“ der linksradikalen Kommunisten, die zum Scheitern der Räterepublik hätten führen müssen (S. 325). Er sieht durchaus die Grenzen des rätedemokratischen Modells, ,die zur Manipulation vieler durch wenige [...] zu degenerieren droht“" (S. 326). Nach dem Übertritt der MSPD ,ins bürgerliche Lager“ und der militärischen Niederschlagung der Räterepublik begann jedoch, so Kuckuk, der „Konsolidierungsprozess der bürgerlichen deutschen Republik“ (S. 333). Diese zwar gründliche ${ }^{34}$, aber unverkennbar von antiparlamentarischen Ressentiments gezeichneten Darstellung fällt gewissermaßen aus

\footnotetext{
32 Kuckuk, Peter (Hrsg.): Revolution und Räterepublik in Bremen, Suhrkamp, Frankfurt a. M. 1969.

33 Ders./Schröder, Ulrich: Bremen in der Deutschen Revolution 1918/1919. Revolution, Räterepublik, Restauration, überarb. und erw. Neuaufl., Edition Falkenberg, Rotenburg/Wümme ${ }^{2} 2017$ (orig. 1986).

34 Kuckuks 1970 bei Fritz Fischer geschriebene Dissertation über Linksradikale und Kommunisten in Bremen von 1918 bis 1920 umfasste fast 700 Seiten.
} 
der Zeit. Zu den aktuellen Fragen an die deutsche Revolution und die Probleme bei der Begründung der ersten Demokratie in Deutschland bietet die Neuauflage keine Anstöße.

Kuckuks stadtgeschichtliche Studie steht damit in deutlichem Gegensatz zu der fundierten und ebenso umfangreichen regionalgeschichtlichen Abhandlung von Wilfried Reininghaus, langjähriger Präsidente des Landesarchivs Nordrhein-Westfalen und ehemaliger Vorsitzender der Historischen Kommission für Westfalen, über die Revolution von 1918/19 in Westfalen und Lippe. ${ }^{35}$ Dem auf den Raum WestfalenLippe bezogenen, überaus fundierten Überblick zum Stand der Forschung über die deutsche Revolution beginnt Reininghaus mit frühen zeitgeschichtlichen Darstellungen und Erinnerungen. In den 1960er Jahren setzte ein breites wissenschaftliches Interesse an den Arbeiter- und Soldatenräten ein. Ortsgeschichten ließen dann die Vielfältigkeit der Räte im Raum Westfalen und Lippe hervortreten. Die weitere historische Forschung hat sich nach Reininghaus auf die spezifischen lokalen, häufig stark voneinander abweichenden Ursachen der Revolution sowie auf die verschiedenen Formen von Räten, von Arbeiter- und Soldaten- bis hin zu Bauern- und Bürgerräten konzentriert. Reininghaus plädiert daher für eine Ausweitung der sozialgeschichtlichen Perspektive. Nicht nur Arbeiter und die Arbeiterbewegung sollten als Träger der Revolution in den Blick genommen werden, sondern auch andere Klassen. Ferner ist die Bedeutung von Frauen in der Revolution in den Fokus zu rücken. Reinhard Rürup zitierend sieht es Reininghaus als dringlich an, die Kräfte der Gegenrevolution und die antidemokratischen Akteure genauer zu untersuchen. Weitere Fragen sind im Sinne einer Kulturgeschichte des Politischen an die Wahlen zu stellen. Hinsichtlich der Aktualität biografischer Ansätze in den Geschichtswissenschaften wäre den Lebenswegen der Akteure der Revolution nachzugehen. Schließlich bezeichnet Reininghaus die Bedeutung des Antisemitismus in der Revolution als ein entscheidendes, bisher vernachlässigtes Forschungsfeld.

Im folgenden Kapitel gibt Reininghaus einen Überblick über die wesentlichen Quellen und Archivalien, wobei der Leser hier in besonderer Weise von den Kompetenzen des ehemaligen Präsidenten des Landesarchivs profitieren kann. Da Reininghaus im Vorwort auf die Notwendigkeit verweist, im Hinblick auf die Einhundertjahrfeier die Bedeutung der Arbeiter- und Soldatenräte zu erfassen, enthält der Band abschließend ein umfangreiches, 122-seitiges Verzeichnis aller bisher dokumentierten Räte von 1918/19 in Westfalen und Lippe mit entsprechenden Archivhinweisen. So bietet diese Publikation eine Fülle von Anregungen für weitere Forschungen, die ebenso für andere Teile des Reiches fruchtbare Hinweise geben.

Die von Reininghaus aufgeworfenen Fragen an die Revolution liegen auch dem von Julia Schafmeister, Bärbel Sunderbrink und Michael Zelle herausgegebenen Band über die Revolution im Fürstentum Lippe zugrunde ${ }^{36}$, der als Begleitband zur

\footnotetext{
35 Reininghaus, Wilfried: Die Revolution 1918/19 in Westfalen und Lippe als Forschungsproblem. Quellen und offene Fragen. Mit einer Dokumentation zu den Arbeiter-, Soldaten- und Bauernräten (Veröffentlichungen der Historischen Kommission für Westfalen, N. F., Bd. 33), Aschendorff, Münster 2016.

36 Schafmeister, Julia/Sunderbrink, Bärbel/Zelle, Michael (Hrsg.): Revolution in Lippe. 1918 und der Aufbruch in die Demokratie (Kataloge des Lippischen Landesmuseums Detmold, Bd. 23/Sonderveröffentlichungen des Naturwissenschaftlichen und Historischen Vereins für das Land Lippe, Bd. 94), Verlag für Regionalgeschichte, Bielefeld 2018.
} 
Ausstellung im Lippischen Landesmuseum erschienen ist. Die Herausgeberinnen Schafmeister und Sunderbrink heben in ihrer Einleitung die Bedeutung der Rätebewegung für die Politisierung der Bevölkerung im Kleinstaat Lippe hervor. Deren Besonderheit bestand in diesem Fürstentum darin, dass hier neben Sozialdemokraten ebenso Liberale in den Räteorganisationen aktiv waren. Diese Koalition, so betonen Schafmeister und Sunderbrink, ,hatte die revolutionäre Übergangsphase erstaunlich gut gemeistert" (S. 17). Als entscheidende Leistung der Revolution heben sie die Durchsetzung eines demokratischen, auch Frauen zuerkannten Wahlrechts für den neuen Freistaat hervor.

Gegliedert in die Kapitel „Revolutionäre Dynamik“, „Akteure der Revolution“, „Revolution in einer Kleinstadt“ und „Institutionen in der Revolution“ gibt der Band neben einem Überblick zum revolutionären Geschehen biografische Porträts, nicht nur von sozialdemokratischen Revolutionären und Frauen in der Revolution, sondern auch von dem letzten Fürsten Leopold IV. Kommunikationswissenschaftliche Fragen greift vor allem die Mitherausgeberin Sunderbrink auf, indem sie der Bedeutung von Medien (Zeitungen, Plakate), Orten (Gastwirtschaften) und Symbolen (Rote Fahnen) nachgeht.

Neben dem westfälischen und lippischen Revolutionsgeschehen galt ein besonderes Interesse der Entwicklung in Bayern, wo die Revolution den dramatischsten, blutigsten und für die Geschichte Deutschlands und Europas folgenreichsten Verlauf nahm. Unter dem irreführenden Titel „Die letzte Nacht der Monarchie. Wie Revolution und Räterepublik in München Adolf Hitler hervorbrachten“ komponiert der Regisseur beim Bayerischen Rundfunk Michael Appel aus Selbstzeugnissen, Augenzeugenberichten und Erinnerungen ein Panorama der Revolution von 1918/19 in Bayern und München. ${ }^{37}$ Seine vornehmlichen Quellen sind die Erinnerungen des als revolutionärer Bohemien vorgestellten Schriftstellers Oskar Maria Graf, die Tagebuchaufzeichnungen des rechtskonservativen Gymnasiallehrers Josef Hofmiller oder die Erinnerungen des Historikers Karl Alexander von Müller, Mitarbeiter der radikalnationalistischen ,Süddeutschen Monatshefte“, der schon vor 1933 als nationalsozialistischer Historiker hervortrat. Appel wollte „kein streng wissenschaftliches“ Buch vorlegen, sondern ,die Menschen zu Wort kommen“ lassen sowie die „emotionalen Erregungszustände“ und den „Schwall der Leidenschaften“ deutlich machen (S. 8f.). Die Auswahl der Zeitzeugen und Quellen reflektiert er jedoch nicht, und seine „Sehepunkte“ legt er ebenso wenig offen. Lediglich im letzten, nur 36 Seiten umfassenden Kapitel seiner 360-seitigen Kompilation kommt Appel zu der im Untertitel angekündigten Hervorbringung von Adolf Hitler aus dem München der Revolutionszeit.

In seinem Schlusswort unter der Überschrift „Wunschdenken“ reduziert Appel die Geschichte, die er aus seinen Quellen zusammengestellt hat, auf die nivellierende Formel, dass das, was die Revolutionäre ebenso wie die Gegenrevolutionäre angetrieben habe, ,geistiger Extremismus“ gewesen sei (S. 350). Sein schlichtes, die historischen Besonderheiten vor allem der antisemitischen und antirepublikanischen

\footnotetext{
37 Appel, Michael: Die letzte Nacht der Monarchie. Wie Revolution und Räterepublik in München Adolf Hitler hervorbrachten, dtv, München 2018.
} 
Gewalt vernebelndes Fazit lautet: ,die Bereitschaft zum Exzess ist der tiefschwarze Schatten, den diese Zeit wirft" (S. 351).

Hatte Appel sich noch bemüht, nachvollziehbar zu argumentieren, so beschränkt sich der ehemalige Gastgeber des „Literarischen Quartetts“ beim Zweiten Deutschen Fernsehen, Volker Weidermann, ganz auf das Erzählerische. Er hat rechtzeitig zum Revolutionsjubiläum ein Buch über die Münchener Räterepublik mit dem Titel: „Träumer. Als die Dichter die Macht übernahmen“ vorgelegt. ${ }^{38}$ Im jovialen Plauderton schreibt Weidermann vom Leben einiger Schriftsteller wie Oskar Maria Graf oder Rainer Maria Rilke. Immer wieder bezieht er sich auf Thomas Mann. Über den Umstand, dass dessen „Betrachtungen eines Unpolitischen“ genau Anfang November 1918 erschienen, schreibt er: ,Das ist wirklich schlecht gelaufen“ (S. 40). Die saloppe Ausdrucksweise findet sich dann auch, wenn er Gustav Landauer neben Kurt Eisner als „,den anderen bärtigen Guru“ (S. 57) abtut. Über die Wahlen zum bayerischen Landtag witzelt er: „Also Wahlen. Die ganze Stadt auf den Beinen, im Sonntagsstaat, verkleidet für die junge Demokratie“ (S. 86). Das Attentat des Mitglieds des Arbeiterrats Alois Lindner auf den Vorsitzenden der SPD und Innenminister Erhard Auer schildert Weidermann im Stil von Groschenromanen: „Lindner rast in Richtung Parlament. Er hat einen Browning in der Faust“ (S. 115). Über die Trauerfeier anlässlich des Mordes an Kurt Eisner, an der annähernd 100.000 Münchnerinnen und Münchner teilnahmen, schreibt Weidermann: „Die politischen Stars dieser Tage fahren in Hofequipagen vor" (S. 130). Nachdem Erich Mühsam sich erfolglos selbst als Volkskommissar für das Auswärtige vorgeschlagen hatte, herrschte, wie Weidermann schreibt, ,verlegenes Schweigen im Schlafzimmer der Königin“, gemeint ist der Sitzungssaal der konstituierenden Sitzung der Räteregierung (S. 151). In diesem Stil geht es weiter: „Dann kommt der 30. April. Es ist aus“ (S. 239). Das Buch ist ganz im Spiegel-Jargon geschrieben. Was Weidermanns Sprache auszeichnet, ist die ,Koketterie mit der eigenen Gewitztheit [...], die eingestreuten Modewörter, der Slang der Saison, [...] dazu eine kleine Zahl syntaktischer Gags“". ${ }^{39}$ Weidermann hat keine klare Fragestellung und keine erkennbaren Erkenntnisziele. Einsichten in die Dramatik der Ereignisse und die Tragik der politisch engagierten Schriftsteller bietet dieses Buch nicht.

Damit unterscheidet sich diese Erzählung fundamental von der fundierten wissenschaftlichen Studie von Michael Brenner über Münchner Juden und Antisemiten in Revolution und Konterrevolution. ${ }^{40}$ Brenner hat eine präzise Fragestellung und kann auf gründliche eigene Archivstudien zugrückgreifen. Die ,jüdische Herkunft vieler Revolutionäre“ war, wie er betont, ,ein heftig diskutiertes Thema“ unter Münchner Jüdinnen und Juden. Diese waren überwiegend ,entschiedene Gegner der Revolution“ oder sahen sie ,zumindest mit Sorge“ (S. 14). Brenner geht es darum, „das

\footnotetext{
38 Weidermann, Volker: Träumer. Als die Dichter die Macht übernahmen, Kiepenheuer \& Witsch, Köln 2017.

39 Enzensberger, Hans Magnus: Die Sprache des Spiegel, in: ders., Einzelheiten I. Bewußtseins-Industrie, Suhrkamp, Frankfurt a. M. 1964, S. 74-105, hier S. 82. Bizarr ist, dass Enzensberger den Band auf dem Umschlag mit der Bemerkung: „Lustig, aufregend, viel Neues, tolle Recherche“ bewirbt.

40 Brenner, Michael: Der lange Schatten der Revolution. Juden und Antisemiten in Hitlers München 1918 bis 1923, Jüdischer Verlag, Berlin 2019.
} 
Geschehen stärker in den Kontext der jüdischen Geschichte" einzubinden (S. 19). Die jüdischen Revolutionäre nahmen, wie er herausarbeitet, am Gemeindeleben kaum mehr teil und hatten keinen Bezug zur Religion. Aber ,sie verleugneten ihr Judentum auch nicht“ (S. 21). Der ,antisemitische Mythos einer, jüdischen Revolution“" ist daher „ebenso absurd wie die Schutzbehauptung der jüdischen Gemeinde, die jüdischen Revolutionäre seien alle keine Juden mehr“ (S. 28).

Didaktisch versiert setzt Brenner seine Intention, die Geschichte der bayerischen Revolution und Konterrevolution stärker in den Kontext der jüdischen Geschichte einzubinden, um, indem er die Kapitel seiner Darstellung jeweils mit Ausführungen über die im zeitlichen Kontext der Ereignisse gefeierten jüdischen Feste einleitet. Er geht detailliert den Beziehungen jüdischer Revolutionäre wie Kurt Eisner, Gustav Landauer, Erich Mühsam, Ernst Toller oder Eugen Leviné zum Judentum nach und fragt, welchen Einfluss ihre Herkunft auf ihre politische Tätigkeit hatte. Daneben beleuchtet er weniger im Zentrum des Geschehens stehende jüdische Akteure sowie eine Reihe von liberalen oder sozialdemokratischen Juden, die die Regierung von Johannes Hoffmann unterstützten, ferner konservative und schließlich rechtsextreme Juden, die gegen die Räterepublik und teils gar in den Freikorps kämpften.

Das Kapitel „Pogromstimmung in München“ thematisiert den unmittelbar nach dem Amtsantritt von Eisner einsetzenden Antisemitismus. Brenner weist dabei auf eine im Bundesarchiv reponierte Sammlung mit hunderten von antisemitischen Hetzbriefen hin, die Eisner zugesandt wurden. Nach der Niederschlagung der Räterepublik mussten sich die Behörden mit dem forcierten Judenhass und der Gefahr von Pogromen auseinandersetzen, wobei die diesbezüglichen Polizeiberichte, wie Brenner zeigt, ,voller antisemitischer Stereotype“ waren (S. 155). München wurde zum „Hort der Reaktion“ und „zur Hauptstadt des Antisemitismus in Deutschland“ (S. 183). In diesem historischen Moment begann Hitlers ,,rasanter politischer Aufstieg“ (S. 192). Der Putsch vom 9. November 1923 wurde für „die meisten Juden Münchens“, so Brenner, ,die erste wirkliche Konfrontation mit dem lebensbedrohlichen Schrecken des nationalsozialistischen Terrors“" (S. 282).

Neben den Studien über München veröffentlichte Frank Jacob, Professor für Globalgeschichte an der Nord Universitet, Norwegen, eine Untersuchung über die Revolution in den unterfränkischen Städten Aschaffenburg, Kitzingen, Schweinfurt und Würzburg. ${ }^{41}$ In Unterfranken habe sich, so sein Ergebnis, die Revolution höchst unterschiedlich entwickelt. An einigen Orten kam es zu einer Eskalation der Gewalt, während die Revolution in anderen Orten friedlich verlief. Es bildete sich eine starke Rätebewegung heraus, doch selbst innerhalb dieser bayerischen Peripherie zeigten die Räte erhebliche Unterschiede. Die Akteure in den unterfränkischen Städten waren, so das Fazit von Jacob, kaum ,,an radikalen Veränderungen interessiert“ (S. 156), sie arbeiteten vielmehr mit den alten Behörden zusammen. In Aschaffenburg wurde zwar eine Räterepublik ausgerufen, doch fehlte es den Akteuren an Unterstützung. In Würzburg gelang es einer kleinen extremen Gruppe, zentrale Orte der Stadt zu besetzen und ebenfalls eine Räterepublik auszurufen. Sozialdemokratie und Ge-

\footnotetext{
41 Jacob, Frank: Revolution und Räterepublik in Unterfranken. Eine landesgeschichtliche Untersuchung zu Verlauf und Folgen der Revolution von 1918/19 an der bayerischen Peripherie, Königshausen \& Neumann, Würzburg 2019.
} 
werkschaften sowie die Beamten der Stadt und die Soldaten lehnten diese jedoch ab. In Würzburg kam es gar zu einer „Bürgerdemonstration gegen die Räterepublik“ (S. 156). In Unterfranken dominierte, wie in anderen Teilen des Reiches, die Furcht vor dem Bolschewismus. Den Akteuren der Rätebewegung ging es um Ruhe und Ordnung. Die unterfränkischen Räte traten nach Jacob für eine demokratische und parlamentarische Demokratie ein.

\section{Die Sozialdemokratie in der deutschen Revolution}

Eines der zentralen Probleme bei der Deutung der deutschen Revolution von 1918/19 ist die Politik der Sozialdemokratie. Im Fokus des Interesses stehen dabei die Spaltung der Arbeiterbewegung sowie die Haltung der Sozialdemokratie zu den Bolschewiki.

Der Bruch der deutschen Sozialdemokratie ging allein auf den Krieg zurück. Der von Uli Schöler und Thilo Scholle herausgegebene Band geht den Folgen dieser aus dem Krieg entstandenen Spaltung für die politische Entwicklung der Revolution nach. ${ }^{42}$ Ziel der Publikation ist es, die herkömmliche, eher aus teleologischen Perspektiven gespeiste und gänzlich überholte dichotomische Sichtweise von der Spaltung der Arbeiterbewegung in zaudernde, die Revolution verratende Reformisten und prinzipientreue Revolutionäre zu überwinden. Diese Darstellung, so betonen die Herausgeber in der Einleitung, wird den historischen Prozessen ebenso wenig gerecht, wie die schematische Gegenüberstellung von demokratischem Parlamentarismus versus Rätediktatur.

Der konzeptionelle Zugang des Bandes ist ein biografischer. Er versammelt 32 Beiträge über bisher eher unterbelichtete Akteure. So zeigt er eine breite Palette unterschiedlicher Einstellungen und eine Vielfalt divergierender politischer Positionen auf, die zugleich die Offenheit der Situation erkennbar machen.

Die Spaltung der deutschen Arbeiterbewegung beschränkte sich indes nicht auf die Partei, sondern schlug sich auf die Gewerkschaften nieder. Zu den entscheidenden gewerkschaftlichen Akteuren gehörten die revolutionären Obleute aus den Betrieben, die wiederum die entscheidenden Initiatoren der Arbeiterräte waren. Dirk H. Müller, seinerzeit Mitarbeiter der Sektion zur Geschichte der Arbeiterbewegung in der einstigen Historischen Kommission zu Berlin, erweiterte aus Anlass der Hundertjahrfeier seine eigenen Studien über die Gewerkschaften vor 1918 auf die Rätebewegung. ${ }^{43}$ Müller verfolgt das politische Handeln der gewerkschaftlichen Aktivisten vom November 1918 bis zum ,,allmählichen Verschwinden der revolutionären Obleute“ in der ersten Hälfte des Jahres 1919. Diese agierten nach Müller bis Oktober 1918 gemäß ,der traditionellen sozialdemokratischen Programmatik“ (S. 319) und waren im November die Initiatoren der Arbeiter- und Soldatenräte. Der Spartakusaufstand 1919 - nach Müller getragen von einer „Mischung aus revolu-

\footnotetext{
42 Schöler, Uli/Scholle, Thilo (Hrsg.): Weltkrieg. Spaltung. Revolution. Sozialdemokratie 1916-1922, Dietz Nachf., Bonn 2018.

43 Müller, Dirk H.: Die revolutionären Obleute und der November 1918. Zur Verschränkung von institutioneller Revolution und Rätebewegung, BoD, Norderstedt 2019.
} 
tionärer Euphorie, unmittelbarer utopischer Zukunftserwartung, Selbstüberschätzung und [...] Unerfahrenheit“ - wurde zum entscheidenden Wendepunkt. Diese Unruhen, in denen ,die revolutionären Obleute mal Aktivisten, mal Vermittler, mal Moderatoren“ waren, schwächten die Gruppe, so Müllers Resümee, „,nachhaltig“ (S. 323). Die revolutionären Obleute hatten versucht, rätedemokratische Vorstellungen in die Industriegesellschaft einzubinden, doch kamen sie nach Müller über ,gedankliche Andeutungen kaum hinaus“. 50 Jahre später diente die Auseinandersetzung mit ihnen, so seine abschließende Bemerkung, nurmehr - Müller bezieht sich hierbei auf Publikationen der 1970er Jahre - der ,,revolutionären Nostalgie“ und als „Basis utopischer Legenden“" (S. 324).

Nicht nur, dass die revolutionären Obleute im Zuge der revolutionären Entwicklung ihre Bedeutung verloren, es vervielfältigten sich die Konfliktlinien innerhalb der Unabhängigen Sozialdemokratie. Der wiederum in der Reihe ,Weimarer Schriften zur Republik“ erschienene und von Andreas Braune, Mario Hesselbarth und Stefan Müller herausgegebene Band über die USPD zwischen Sozialdemokratie und Kommunismus bietet eine Bestandsaufnahme 100 Jahre nach der Gründung der Partei. ${ }^{44}$ Er versammelt Beiträge zu der zwischen einer Rückkehr zur Sozialdemokratie und einer Ausrichtung auf den Bolschewismus zerrissenen USPD. Darüber hinaus sind dem Band 18 Quellen zur Geschichte dieser Partei beigegeben.

Ein Blick auf die Unabhängige Sozialdemokratie, so die Herausgeber, ,schützt vor teleologischen, binären oder simplifizierenden Sichtweisen“ und zeigt, dass „,der Zukunftshorizont für die Akteure in jedem Moment" offen war (S. X). Die Einleitung geht zunächst den Ursachen der Spaltung im Krieg und der Burgfriedenspolitik der SPD nach. Die USPD führte einst verfeindete sozialdemokratische Politiker zusammen, zugleich prallten in ihr, wie die Herausgeber betonen, unterschiedliche Erwartungsräume und abweichende Zukunftsvorstellungen aufeinander. Nach dem November 1918 wurde diese im und gegen den Krieg gegründete Partei ,die eigentliche Partei der Revolution“, der es um Sozialisierung, Zerschlagung der alten Eliten und um den Versuch ging, die anvisierte parlamentarische Demokratie mit rätedemokratischen Elementen zu ergänzen.

Wie der Beitrag von Mike Schmeitzner über Karl Kautsky und seine scharfe Kritik an Lenins Diktatur des Proletariats in diesem Band deutlich macht, waren deutsche Sozialdemokraten beider Richtungen über die diktatorische Entwicklung in Russland nach dem Oktoberputsch der Bolschewiki sehr genau im Bilde. Und sie waren sich klar darüber, dass die russischen Sozialdemokraten zu den ersten Opfern des bolschewistischen Terrors gehörten.

Einige dieser Beiträge von Karl Kautsky finden sich neben weiteren Artikeln unabhängiger Sozialdemokraten zur bolschewistischen Revolution in einer von Jörn Schütrumpf herausgegebenen Quellenedition. Schütrumpf hat über 100 Beiträge dieser breiten, in der Zeit zwischen dem Putsch der Bolschewiki in Russland und der deutschen Revolution geführten Diskussion zusammengestellt. ${ }^{45}$ Publiziert wur-

\footnotetext{
44 Braune, Andreas/Hesselbarth, Mario/Müller, Stefan (Hrsg.): Die USPD zwischen Sozialdemokratie und Kommunismus 1917-1922 (Weimarer Schriften zur Republik, Bd. 3), Steiner, Stuttgart 2018.

45 Schütrumpf, Jörn (Hrsg.): Diktatur statt Sozialismus. Die russische Revolution und die deutsche Linke 1917/18, Dietz, Berlin 2017.
} 
den sie vor allem in Zeitschriften wie dem ,Sozialdemokrat“ aus Stuttgart, einem Blatt der Spartakusgruppe, oder der „Sozialistischen Auslandspolitik“, dem Organ der sozialdemokratischen Kriegsgegner. Ein Teil der deutschen Linkssozialisten trat unverkennbar für einen diktatorischen Weg ein, wie ihn die Bolschewiki eingeschlagen hatten, die Mehrheit aber lehnte die bolschewistische Politik der Gewalt und des Terrors ab.

Die Kritik an der Politik Lenins teilten die einst heftig verfeindeten Antipoden der Vorkriegssozialdemokratie, Eduard Bernstein und Karl Kautsky, die politisch und persönlich bereits durch die gemeinsame Ablehnung des Krieges wieder zueinander gefunden hatten und gemeinsam an der Gründung der USPD beteiligt waren. Vielversprechend hinsichtlich der Wahrnehmung der bolschewistischen Politik in Russland war daher der von Eva Bettina Görtz herausgegebene Briefwechsel zwischen Eduard Bernstein und Karl Kautsky aus den Jahren 1912 bis 1932. ${ }^{46}$ Nachdem deren Kommunikation im Jahr 1901 in Folge der erbitterten Auseinandersetzung über den Revisionismus abgebrochen war, nahmen sie kurz vor dem Krieg ihre Korrespondenz wieder auf. Nach dem Beginn des Krieges verband sie die klare Kritik an den nationalistischen Positionen, die in der SPD vertreten wurden. Da beide in den Jahren von Krieg und Revolution in Berlin lebten, war die Korrespondenz jedoch in dieser Zeit dünn, und für die hier interessierende Zeit von November 1918 bis März 1921 liegen bedauerlicherweise gar keine Briefe vor. Politische Verstimmungen oder Divergenzen aber waren für diese Lücke, wie die Herausgeberin in ihrer Einleitung schreibt, nicht verantwortlich. Mit dem Umzug von Karl und Luise Kautsky nach Wien wurden die Korrespondenzen ab 1924 wieder umfangreicher, und sie zeigten nun zwei verschiedene Schwerpunkte der beiden immer stärker ins Abseits gedrängten Sozialdemokraten. Während sich Bernstein intensiv um eine Aufarbeitung der Schuld des Deutschen Kaiserreiches am Ersten Weltkrieg bemühte, stand für Kautsky die Kritik am Bolschewismus im Vordergrund. Darüber hinaus engagierte sich vor allem Bernstein im Kampf gegen den Antisemitismus der völkisch-nationalistischen Bewegung. In den Korrespondenzen schlägt sich dies jedoch ebenso wenig nieder wie deren Auseinandersetzung mit dem Zionismus und Bernsteins verstärkte Hinwendung zu Fragen des Judentums.

\section{Bürgerliche Intellektuelle und die Revolution}

Der Fokus der geschichtswissenschaftlichen Studien über die Revolution sowie eines erheblichen Teils der neueren Gesamtdarstellungen liegt vor allem auf den verschiedenen Protagonisten der Arbeiterbewegung. Obgleich vielzitierte Tagebuchaufzeichnungen und Erfahrungsberichte zeitgenössischer Beobachter aus dem Bürgertum - etwa Thomas Mann, Viktor Klemperer, Oskar Maria Graf oder Harry Graf Kessler - über die Revolutionstage vorliegen, sind Neuerscheinungen von EgoDokumenten bürgerlicher Intellektueller aus der Zeit der Revolution rar. Umso begrüßenswerter ist es daher, dass die zeitgenössischen Kommentare und kritischen

46 Görtz, Eva Bettina (Hrsg.): Eduard Bernsteins Briefwechsel mit Karl Kautsky (1912-1932), Campus, Frankfurt a. M./New York 2011. 
Beobachtungen des protestantischen Theologen, Kulturkritikers und liberalen Politikers Ernst Troeltsch, die dieser zunächst unter dem Titel „Spectator-Briefe“, dann „Berliner Briefe“, zwischen 1919 und 1922 in der Zeitschrift „Kunstwart“ veröffentlichte, nun in einer vorzüglichen, von Gangolf Hübinger betreuten Ausgabe im Rahmen der „Kritischen Gesamtausgabe“ der Werke von Troeltsch vorliegen. ${ }^{47}$

Troeltsch schrieb diese Artikel auf der Grundlage eigener Beobachtungen, einer genauen Zeitungslektüre und vielfältiger Gespräche mit verschiedenen Zeitgenossen und Akteuren der revolutionären Bewegung und kommentierte so aufmerksam den Verlauf der revolutionären Entwicklung aus einer linksliberalen Perspektive. ${ }^{48}$ In seinen „Spectator“-Briefen suchte er die Dynamik und Widersprüchlichkeit der Umbruchszeit und die Zerrissenheit der deutschen Gesellschaft zu erfassen. Nachdrücklich trat er für eine Zusammenarbeit von Sozialdemokratie und bürgerlichen Liberalen ein und warnte eindringlich vor dem Bolschewismus. Der SPD hielt er zugute, dass sie die Gefahr eines kommunistischen Umsturzes verhindert habe. Hellsichtig warnte Troeltsch vor der Gefahr einer ,deutsche[n] Faszistenbewegung [sic]“, die eine „Ära politischer Morde“ bedeuten würde (S. 446).

Obgleich Troeltsch sich als „Vernunftdemokrat“ bezeichnete (S. 20) und energisch für die neue deutsche Republik eintrat, teilte er die Schuldabwehr des deutschen Bürgertums. So bezeichnete er in einem Brief den „Glauben an die deutsche Schuld“ als „die ganz große Weltsuggestion“ (S. 118). Ferner blieb er dem antiwestlichen Denken des Wilhelminischen Kaiserreichs insofern verhaftet, als er nach der Niederlage des Deutschen Kaiserreiches von dem „Weltsystem der Entente“ als „der neuen, zum Schutz der Kriegsgewinne geschaffenen heiligen Allianz“ sprach (S. 363). In dieser erblickte er einen Sieg des Amerikanismus (S. 484). In einem weiteren Aspekt blieb Troeltsch Motiven des Kulturpessimismus des Kaiserreichs treu, so wenn er - obwohl er den politischen Antisemitismus des neuen Nationalismus klar verurteilte - in seinem „Vorherrschaft des Judentums?“ überschriebenen Brief selbst antisemitische Denkmuster übernahm (S. 209-217).

Ein Tableau der am revolutionären Geschehen beteiligten Intellektuellen geben Heidi und Wolfgang Beutin. ${ }^{49}$ Neben einer Gruppe von beobachtenden Künstlern unterscheiden sie bürgerliche Intellektuelle in demokratischen Parteien von Intellektuellen in der Arbeiterbewegung. So porträtieren sie etwa intellektuelle Akteure in der pazifistischen Bewegung, im Spartakusbund und in der Rätebewegung.

Während Beutin und Beutin sich auf stichwortartige Kurzporträts von Intellektuellen ergänzt durch kurze Auszüge aus deren Schriften beschränken, präsentiert die Publikation von Albert Dikovich und Alexander Wierzock, wiederum in der Schriftenreihe „Weimarer Schriften zur Republik“ erschienen und aus einer Tagung an der Universität Wien hervorgegangen, die Visionen von Philosophen, Human-

\footnotetext{
47 Troeltsch, Ernst: Kritische Gesamtausgabe, Bd. 14: Spectator-Briefe und Berliner Briefe (1919-1922), hrsg. v. Gangolf Hübinger, De Gruyter, Berlin 2015.

48 Nach einer ersten posthum erschienenen, gekürzten und von Hans Baron zusammengestellten und herausgegebenen Ausgabe im Jahr 1924 besorgte Johann Hinrich Claussen 1994 eine neue Edition in der „Anderen Bibliothek“, die jedoch ebenfalls unvollständig war: Troeltsch, Ernst: Die Fehlgeburt einer Republik. Spektator in Berlin 1918-1922, Die Andere Bibliothek, Frankfurt a. M. 1994.

49 Beutin, Heidi/Beutin, Wolfgang: Fanfaren einer neuen Freiheit. Deutsche Intellektuelle und die Novemberrevolution, WBG, Darmstadt 2018.
} 
wissenschaftlern und Schriftstellern über den „Neuen Menschen“ im Mitteleuropa nach dem Ersten Weltkrieg. ${ }^{50}$ Der Band bietet überraschende Einblicke in die $\mathrm{Zu}$ kunftsentwürfe liberaler oder sich selbst eher als unpolitisch einschätzender Wissenschaftler. Darüber hinaus zieht er einen ideengeschichtlichen Bogen von den intellektuellen Konstellationen der zwischen utopischen Lebensreformen und Fin de Siècle-Stimmung zerrissenen Zeit um 1900 zum neuen Aufbruch nach Kriegsende, und er bezieht Protagonisten aus dem Raum der ehemaligen Habsburgermonarchie ein; er macht mithin deutlich, dass die im Zuge der revolutionären Entwicklung sich verstärkende Diskussion über die Verbesserung des Menschen eine europäische Debatte war.

\section{Die Musealisierung der Revolution im Centenaire}

Wie sehr nicht nur Schriftsteller, sondern auch bildende Künstler an diesem Aufbruch und der Suche nach dem „Neuen Menschen“ teilhatten, zeigt der von Thomas Köhler, Ralf Burmeister und Janina Nentwig herausgegebene Ausstellungskatalog über die Anfang Dezember 1918 gegründete Gruppe von Malern, Bildhauern und Architekten, die sich programmatisch unter dem Namen „Novembergruppe“ zusammenfanden. Anfangs gehörten ihr etwa 170 Künstler, darunter etwa Max Pechstein, César Klein, Georg Tappert oder Georg Leschnitzer, und einige wenige Künstlerinnen an. ${ }^{51}$

Im Januar 1919 veröffentlichte die Gruppe ein programmatisches Manifest, in dem sie für eine umfassende Demokratisierung der Kunst und Kulturpolitik eintrat. Dezidiert engagierte sie sich für den Aufbau der Republik. Während die Kunst der Revolutionszeit und der Weimarer Republik vielfach mit Akteuren der extremen Linken assoziiert wird, trat die Novembergruppe unmissverständlich für die parlamentarische Demokratie ein. So arbeitete sie mit der Abteilung für politische Bildung des Arbeiter- und Soldatenrats und des Rats der Volksbeauftragten zusammen. Für diesen „Werbedienst“ zum Aufbau der parlamentarischen Demokratie schufen Mitglieder der Novembergruppe eine Reihe von Plakaten, in denen sie „Arbeiter, Bürger, Bauern, Soldaten “ dazu aufriefen, sich für die Nationalversammlung einzusetzen. Ein anderes Plakat warnte davor, die ,junge Freiheit“ nicht „durch Unordnung und Brudermord“ zu erwürgen, ein weiteres rief die Arbeiter dazu auf, nicht durch Streiks die Ernährung zu gefährden: „Streik zerstört, Arbeit ernährt“. Die Künstler der Novembergruppe wurden, wie Kristina Kratz-Kessemeier in ihrem Essay festhält, „Größen einer selbstbewussten modernen Kunstpolitik der sich festigenden Republik“" (S. 35).

Das Museum für Fotografie in Berlin zeigte zum Einhundertjährigen der Revolution aus den umfangreichen Beständen der Berliner Kunstbibliothek die Ausstellung

\footnotetext{
${ }^{50}$ Dikovich, Albert/Wierzock, Alexander (Hrsg.): Von der Revolution zum Neuen Menschen. Das politische Imaginäre in Mitteleuropa 1918/19. Philosophie, Humanwissenschaften und Literatur (Weimarer Schriften zur Republik, Bd. 5), Steiner, Stuttgart 2018.

${ }^{51}$ Köhler, Thomas/Burmeister, Ralf/Nentwig, Janina (Hrsg.): Freiheit. Die Kunst der Novembergruppe 1918-1935, Prestel, München 2018.
} 
„Berlin in der Revolution 1918/19“, die nicht nur den von den Pressefotografen genauestens dokumentierten Verlauf der revolutionären Entwicklung mit ihren Massendemonstrationen und Straßenkämpfen vom November 1918 bis zum Februar 1919, sondern ebenso die gleichzeitig sich entfaltende Unterhaltungskultur und die Entwicklung der Filmindustrie präsentierte. Die Demokratisierung, so heben die Her ausgeber des Katalogbandes Ludger Derenthal, Evelin Förster und Enno Kaufhold in ihrer Einleitung hervor, ,verlangte nicht allein nach politischer, sondern zugleich nach kultureller Partizipation“ (S. 21). ${ }^{52}$ In seinem Essay „Die revolutionären ,Arbeiter und Soldaten “" kommt der Mitherausgeber Kaufhold nach einer detaillierten Bildanalyse zu frappierenden Einsichten über die Akteure der Revolution. Durch eine genaue Interpretation des Habitus und der Kleidung der abgelichteten Personen kann Kaufhold überzeugend nachweisen, wie wenig die in den schriftlichen Quellen und geschichtswissenschaftlichen Darstellungen durchgängig sich findende Zuschreibung der Revolution als einer ,proletarischen“ zutrifft. Auf nahezu allen Aufnahmen von Massenaufmärschen, Demonstrationen oder Trauerumzügen findet sich neben den Arbeitern eine Vielzahl von bürgerlichen oder kleinbürgerlichen Akteuren, und dies gilt ebenso für die Fotografien der spartakistischen Demonstrationen und Kundgebungen. „Das Spektrum der politisch links orientierten [...] Berliner reichte weit in die Kreise der Angestellten und der kleinbürgerlich-bürgerlichen Schichten hinein“"(S. 75).

\section{Resümee}

Neue gesellschaftliche Erfahrungen und veränderte politische Konfliktlagen in der Gegenwart lassen die Vergangenheit in einem neuen Licht erscheinen. Schon 1929 hatte der amerikanische Philosoph George Herbert Mead in seinen geschichtsphilosophischen Betrachtungen festgehalten, dass jede Generation ihre Geschichte neu schreibt und andere Fragen an die Vergangenheit richtet. Das Bild der Vergangenheit entsteht nach Mead notwendigerweise ,aus der Sicht des neuen Problems von heute" ${ }^{53}$ In diesem Licht soll abschließend versucht werden, in sechs Punkten ein Resümee zu ziehen.

1. Die Bedrohung der Demokratie in den ersten Dekaden des 21. Jahrhunderts führt in einer paradoxen Volte dazu, dass die junge bedrohte Demokratie in den ersten Dekaden des 20. Jahrhunderts nun nicht mehr a priori als zum Scheitern verurteilt gesehen wird. 100 Jahre nach der Revolution treten vielmehr deren Errungenschaften und die Potenziale der Demokratisierung hervor. Für Wolfgang Niess war die Revolution daher, so der Untertitel seiner Gesamtdarstellung, der ,wahre Beginn unserer Demokratie“. 54

\footnotetext{
52 Derenthal, Ludger/Förster, Evelin/Kaufhold, Enno (Hrsg.): Berlin in der Revolution 1918/1919. Fotografie, Film, Unterhaltungskultur, Kettler, Dortmund 2018.

${ }^{53}$ Mead, George Herbert: Das Wesen der Vergangenheit, in: ders., Gesammelte Aufsätze, Bd. 2, Suhrkamp, Frankfurt a. M. 1983, S. 337-346, hier S. 344f.

${ }^{54}$ Niess: Revolution (wie Anm. 18).
} 
Obgleich gelegentlich noch die alten Dichotomien und die Legenden vom sozialdemokratischen Verrat fortgeschrieben werden, so vor allem in den hier vorgestellten Büchern von Bernd Langer und Klaus Gietinger, arbeiten die neuen Gesamtdarstellungen ebenso wie die besprochenen regionalgeschichtlichen Studien die Leistungen und Errungenschaften des revolutionären Umbruchs nach dem November 1918 heraus. Der neue Blick auf die Revolution zeigt somit, dass die alten Legenden von der Revolution als einer „,verratenen“ oder „überflüssigen“ der historischen Urteilskraft nicht standzuhalten vermögen. Wie Alexander Gallus in seinem Beitrag für den Band ,Weimar als Herausforderung“ betonte, ist ,die ebenso alte wie ermüdende Frage nach den verpassten Chancen“ überwunden. ${ }^{55}$ In diesem Sinne kritisiert Robert Gerwarth die in der älteren Historiografie immer wieder verwendeten Zuschreibungen der Revolution. Gerade der in der neueren Forschung noch eher unterbelichtete Vergleich mit anderen europäischen Revolutionen zeigt, wie Gerwarth ebenfalls herausstreicht, dass die Entwicklung in Deutschland ,bemerkenswert erfolgreich“ war.

Der 2018 verstorbene Nestor der (west-)deutschen Revolutionsforschung Reinhard Rürup, der mit seinen Beiträgen aus sechs Jahrzehnten - sie reichen von 1969 bis 2013 - immer wieder neue Anregungen für die Forschung und neue Einsichten in das Revolutionsgeschehen geliefert hat, konnte kurz vor seinem Tod noch eine Auswahl seiner Revolutionsstudien für eine Publikation zusammenstellen. Diese ist nunmehr mit einem Nachwort von Peter Brandt und Detlef Lehnert im Wallstein Verlag erschienen. Am Ende seiner Einleitung plädierte Rürup vehement dafür, dass der Revolution von 1918/19 „ein Ehrenplatz in der deutschen Demokratiegeschichte gebührt" ${ }^{\text {. }} 6$

2. Die Probleme der Gegenwart, aus denen heraus das Bild der Vergangenheit neu erschaffen wird, beschränken sich aber nicht allein auf die Bedrohung der Demokratie durch autoritäre Demagogen und illiberale Politikformen. Sie sind Folge unzureichender demokratischer Teilhabe und mangelnder Partizipationsmöglichkeiten. In diesem Sinne erhält die Frage nach den Räten Aktualität, und dies jenseits der alten Dichotomie von parlamentarischer Demokratie versus Rätediktatur. Anknüpfend an die eingehenden Überlegungen von Rürup über die Räte geht es dabei vor allem darum, die zeitgenössischen politischen Kontroversen über die Aufgaben und Ziele der Räte unter demokratietheoretischen Aspekten neu zu diskutieren. ${ }^{57}$ Relevant wird die Frage der Räte aus aktueller Perspektive nicht zuletzt hinsichtlich der Defizite der gegenwärtigen repräsentativen Demokratie. Der französische Historiker und Politikwissenschaftler Pierre Rosanvallon, derzeit einer der, so Lutz Raphel, „originellsten Theoretiker“ der Demokratie ${ }^{58}$, hat

\footnotetext{
55 Gallus, Alexander: Auf dem Weg zur Reaktualisierung durch Historisierung. Die vergessene Revolution von 1918/19 revisited, in: Dreyer/Braune (Hrsg.): Weimar (wie Anm. 10), S. 9-22, hier S. 18.

56 Rürup, Reinhard: Revolution und Demokratiegründung. Studien zur deutschen Geschichte 1918/19, mit einem Nachwort v. Peter Brandt/Detlef Lehnert, Wallstein, Göttingen 2020, S. 18.

57 Einleitung, in: ders. (Hrsg): Arbeiter-und Soldatenräte im rheinisch-westfälischen Industriegebiet. Studien zur Geschichte der Revolution 1918/19, Hammer, Wuppertal 1975, S. 7-38.

58 Raphael, Lutz: Demokratiegeschichte als Problemgeschichte und Gegenwartsanalyse. Das Werk Pierre Rosanvallons, in: Neue Politische Literatur 58 (2013), H. 1, S. 7-20.
} 
sich vornehmlich am Beispiel Frankreichs und der USA in einen umfangreichen Zyklus mit den Aporien demokratischer Herrschaftsformen und ihren pathologischen Einbrüchen auseinandergesetzt. In seinem 2017 in deutscher Übersetzung erschienenen Werk über „Politik im Zeitalter des Misstrauens“ geht es ihm um die „Geburtsfehler der parlamentarischen Regierungsform“, und er untersucht unter dem eher irritierenden - wenn nicht missverständlichen - Titel „Gegen-Demokratie" die in einer repräsentativen Demokratie wirksamen und notwendigen Instanzen von Kontrolle, Überwachung und Korrektur. ${ }^{59}$ Ziel dieser Gegenmächte ist es, das erforderliche Misstrauen der Regierten gegenüber den von ihnen gewählten Regierenden aufzuwiegen. Rosanvallon hat jedoch in diesem, wiederum vornehmlich auf die französische und amerikanische Geschichte bezogenen Band die dramatische Begründung der deutschen Demokratie nach dem Ersten Weltkrieg ebenso wenig berücksichtigt wie die Frage, ob Arbeiter- und Soldatenräte demokratietheoretisch als eine Instanz genau dieser Kontrolle und Überwachung der repräsentativen Demokratie hätten dienen können. Angesichts des Selbstverständnisses der Arbeiter- und Soldatenräte als Kontrollorgan wäre eine entsprechende demokratietheoretische Reflexion ebenso erhellend wie die Frage, inwiefern die Räte als Moment einer zivilgesellschaftlichen Erweiterung der repräsentativen Demokratie hätten fungieren können.

Diesen Gedanken führt der Historiker Paul Ginsborg in seinem anregenden Essay „Wie Demokratie leben“ aus, in dem er fragt, ob nicht die Rätedemokratie - Ginsborg bezieht sich hierbei auf die russische Rätebewegung vor dem Putsch der Bolschewiki - als „Experiment partizipativer Demokratie“ verstanden werden müsse und ob die Räte zur „Einübung in demokratische Verfahrensweisen“ dienten. ${ }^{60}$ Zur Umsetzung dieses Programms kam es jedoch nicht, da die Räte im Zuge des Aufbaus der bolschewistischen Herrschaft entmachtet wurden und die Partei ihre Alleinherrschaft ausbaute.

Welche Vorstellung die Akteure der Räterepubliken in Bremen, München, Leipzig und Gotha von der Rolle und Bedeutung der Räte hatten sowie die Ursachen von deren Scheitern untersucht Mike Schmeitzner in seinem Beitrag zu dem Band „Weltkrieg. Spaltung. Revolution“. Die Räterepublik, so Schmeitzners Ergebnis, „,blieb in Deutschland eine singuläre und vor allem äußerst fragile Erscheinung, die nicht nur aufgrund der äußeren militärischen Bedrängnis scheiterte“. Im Gegensatz zu Russland war in der deutschen Arbeiterbewegung ,die Tradition des Parlamentarismus zu stark ausgeprägt", sodass diktatorische Tendenzen keinen Rückhalt hatten. ${ }^{61}$

Die Frage nach dem demokratischen und zivilgesellschaftlichen Potenzial der Räte wird indes kontrovers diskutiert, wie nicht zuletzt die These von Hans Rudolf

\footnotetext{
59 Rosanvallon, Pierre: Die Gegen-Demokratie. Politik im Zeitalter des Misstrauens, aus dem Frz. v. Michael Halfbrodt, Hamburger Edition, Hamburg 2017 (frz. 2006).

60 Ginsborg, Paul: Wie Demokratie leben, aus dem Ital. v. Friederike Hausmann, Wagenbach, Berlin 2008 (ital. 2006), hier S. 21.

61 Schmeitzner, Mike: Die Räterepublik als Diktatur des Proletariats. Linksparteien und Regionalentwicklungen in Bremen, München, Leipzig und Gotha 1918/19 im Vergleich, in: Schöler/Scholle (Hrsg.): Weltkrieg (wie Anm. 42), S. 166-179, hier S. 179.
} 
Wahl über den quasiständischen Charakter des Bremer Arbeiterrats zeigt. Nach Wahl erweist sich „das parlamentarische System“ mit seinen allgemeinen Wahlen als „,weitaus partizipativer“ und ,integrativer“ „,als die Herrschaft der Räte“. ${ }^{62}$

Der Komplexität des Gegenstandes entspricht die Vielfalt der zeitgenössischen Debatten. So nahm etwa der Neukantianer Paul Natorp, der für eine Zusammenarbeit zwischen der sozialistischen Arbeiterbewegung und der Gelehrtenrepublik plädierte, das Thema Räte auf, um damit an das Platonische Ideal der Philosophenherrschaft anzuknüpfen. ${ }^{63}$

Jenseits dieser ideengeschichtlichen Betrachtungen zeigt das beeindruckende Verzeichnis von 1241 lokalen Räteorganisationen von 1918/19, die Wilfried Reininghaus allein für den Raum Westfalen und Lippe archivalisch erfasst hat, die realgeschichtliche Breite und Vielfalt der Rätebewegung. ${ }^{64}$

3. Die Anregung von Wilfried Reininghaus für eine sozialgeschichtliche Erweiterung der Räteforschung gilt gleichermaßen für die soziale Zusammensetzung der Teilnehmer von Massenkundgebungen und Aufmärschen sowie der Aktivisten der Konterrevolution und die Mitglieder der Freikorps. So konnte Enno Kaufhold aufgrund seiner detaillierten Bildanalyse von Fotografien der Demonstrationen und Trauerumzüge zeigen, dass neben Arbeitern auch Bürger und Kleinbürger am Revolutionsgeschehen in erheblichem Maße teilnahmen. Die „Vorstellung von einer proletarischen Revolution“, so Kaufholds These, lässt sich „,nicht aufrechterhalten" ${ }^{65}$ Jan-Philipp Pomplun wiederum hat aufgrund einer systematischen Durchsicht der Mitgliedsrollen von Freikorps nachgewiesen, dass die alte Vorstellung, vor allem Studenten und ehemalige Offiziere seien in den Freikorps aktiv gewesen, revidiert werden muss. „Das Gros der Freikorpskämpfer rekrutierte sich aus Handwerksgesellen und Arbeitern. "66

4. Der Umbruch der Revolution in Russland zwischen Februar und Oktober 1917 und der Ausbau des bolschewistischen Terrorregimes ist in Deutschland sehr genau wahrgenommen worden. Die Sorge der deutschen Sozialdemokratie vor einer bolschewistischen Entwicklung war nicht nur Folge einer „blöde[n] Bolschewismusfurcht", von der der unabhängige Sozialdemokrat Wilhelm Dittmann sprach. ${ }^{67}$ Robert Gerwarth macht eindringlich klar, dass diese Angst beileibe nichts Pathologisches an sich hatte. Wie Mark Jones zeigt, kannten die in Russland eingesetz-

\footnotetext{
62 Wahl, Hans Rudolf: Novemberrevolution, Räterepublik und Demokratiegründung in Bremen, in: Lehnert (Hrsg.): Revolution (wie Anm. 30), S. 177-240, hier S. 237.

${ }^{63}$ Dikovich, Albert: Paul Natorps Sozialidealismus. Transpolitisches Regieren im Rätestaat, in: ders./ Wierzock (Hrsg.): Revolution (wie Anm. 50), S. 123-157.

${ }^{64}$ Reininghaus: Revolution (wie Anm. 35), S. 259-379.

${ }^{65}$ Kaufhold, Enno: Die revolutionären „Arbeiter und Soldaten“ in: Derenthal/Förster/Kaufhold (Hrsg.): Berlin (wie Anm. 52), S. 61-76, hier S. 75.

${ }^{66}$ Pomplun, Jan-Philipp: Die Entstehung der Freikorps in der Revolution von 1918/19, in: Zeitschrift für Geschichtswissenschaft 66 (2018), H. 10, S. 813-825, hier S. 822; ders.: Keimzellen des Nationalsozialismus. Sozialgeschichtliche Aspekte und personelle Kontinuitäten südwestdeutscher Freikorps, in: Schmidt, Daniel/Sturm, Michael/Livi, Massimiliano (Hrsg.): Wegbereiter des Nationalsozialismus. Personen, Organisationen und Netzwerke der extremen Rechten zwischen 1918 und 1933 (Schriftenreihe des Instituts für Stadtgeschichte. Beiträge, Bd. 19), Klartext, Essen 2015, S. 73-88, hier v. a. S. $66 \mathrm{ff}$.

${ }^{67}$ Diese Aussage Dittmanns hat Gietinger zitiert in: Gietinger: November 1918 (wie Anm. 23), S. 70.
} 
ten Soldaten „die Realität des Bolschewismus und des russischen Bürgerkrieges aus nächster Nähe“. ${ }^{68}$ Jones wies in diesem Kontext auf die Studien von Georges Lefèbvre über „die große Furcht“ in der Französischen Revolution und dessen Begriff der Autosuggestion hin, die er für das Verständnis der ,großen Furcht“ vor den Bolschewiki in den Jahren 1918/19 fruchtbar gemacht hat. Die unentwegte Wiederholung der Bilder von der bolschewistischen Gewalt wurde so zu einem festen Bestandteil des politischen Weltbildes zahlreicher deutscher Zeitgenossen. Nicht zuletzt die sozialdemokratischen Revolutionäre in Deutschland, wie vor allem die intensive Auseinandersetzung von Karl Kautsky mit der Entwicklung in Russland beweist, waren sich sehr genau darüber im Klaren, dass die russischen Sozialdemokraten zu den ersten Opfern des bolschewistischen Terrors gehörten.

5. Die Frage, wie es möglich war, dass die anfangs friedliche Revolution in einen von exzessiver Gewalt gezeichneten Bürgerkrieg umschlagen konnte, hängt somit nicht zuletzt mit dieser ,großen Furcht“ vor der als drohend wahrgenommenen bolschewistischen Gewaltherrschaft zusammen. Obgleich „,die reale Macht der Spartakusgruppe eher gering war“, wie Robert Gerwarth noch einmal hervorhebt, machte „das Beispiel der russischen Revolution doch sehr anschaulich, dass es lediglich einer kleinen Gruppe entschlossener Berufsrevolutionäre bedurfte, um die Regierung zu übernehmen“" ${ }^{69}$ In diesem Sinne bekräftigt Mark Jones, dass Karl Liebknecht zwar weder im Arbeiter- und Soldatenrat noch im Rat der Volksbeauftragten eine Rolle spielte, „die Machtübernahme Liebknechts“, für die Zeitgenossen aber trotzdem ,eine reale und konkrete Möglichkeit“ darstellte. ${ }^{70}$

Damit ist das Problem der Gewalt jedoch noch nicht gelöst, denn die schwierigere, noch immer nicht befriedigend geklärte Frage ist, wieso die verantwortlichen sozialdemokratischen Politiker im Moment der Eskalation der Gewalt auf das Militär und auf neu aufgestellte paramilitärische Verbände setzen konnten, deren Ziel nicht der Schutz der Republik, sondern deren Bekämpfung war.

Neben der Niederschlagung des Januar- und Märzaufstandes in Berlin nahm die Gewalt vor allem bei der Zerschlagung der Münchner Räterepublik verhängnisvolle Formen an. „Die Grausamkeiten, die dabei geschahen“, so Michael Appel in seiner Darstellung, „lassen immer wieder Atem und Stimme stocken““.71

Daher macht es sich Joachim Käppner zu leicht, wenn er erklärt, dass die SPD die Rechten unterschätzt und die radikalen Linken überschätzt habe, oder wenn er über Gustav Noske schreibt, dass dieser mit „,der historischen Rolle“ heillos überfordert war. ${ }^{72}$ Auch Wolfgang Niess macht es sich zu einfach, wenn er schreibt, dass das Bündnis der sozialdemokratischen Regierung mit der alten kaiserlichen Armeeführung unnötig gewesen ist, so richtig seine These ist, dass die verschwörungstheoretischen Deutungen vom „Ebert-Groener-Pakt“ ins „Reich der Legenden“" gehören. ${ }^{73}$

\footnotetext{
68 Jones: Anfang (wie Anm. 24), S. 22.

69 Gerwarth: Revolutionen (wie Anm. 17), S. 204.

70 Jones: Anfang (wie Anm. 24), S. 79.

71 Appel: Nacht (wie Anm. 37), S. 295.

72 Käppner: 1918. Aufstand (wie Anm. 19), S. 456.

73 Niess: Revolution (wie Anm. 18), S. 15.
} 
Mit dem Bürgerkrieg zog der Krieg in die zuvor von Zerstörungen gänzlich verschonten deutschen Städte ein. In diesem Kontext wurden etwa, wie Mark Jones zeigt, die Legenden von Franktireurs, wie sie bei der deutschen Besetzung Belgiens im August 1914 unter deutschen Soldaten zirkulierten, wieder lebendig. Nun richteten sie sich nicht gegen die belgische, sondern gegen die deutsche Zivilbevölkerung. ${ }^{74}$

Das Vertrauen, das Teile der sozialdemokratischen Handlungsträger in das deutsche Heer hatten, war eng verbunden mit deren Verweigerung, die Verantwortung des deutschen Reiches und seines Militärs für den Krieg aufzuarbeiten. Diese Schuldabwehr - die auch von Teilen der SPD getragen war - führte zur Blindheit gegenüber dem deutschen Militär, die wiederum das Verständnis für die von diesem propagierten ,soldatischen Nationalismus“ und seines extremen Antisemitismus versperrte. ${ }^{75}$

6. Das Thema Antisemitismus ist in den neueren Studien und Gesamtdarstellungen zur deutschen Revolution keineswegs ausgeblendet, und doch bleibt es vielfach im Unklaren, wie es möglich war, dass sich am Beginn des Zeitalters der Extreme ein extremer Antisemitismus herausbilden konnte. Wilfried Reininghaus hat in seinem problemgeschichtlichen Aufriss darauf hingewiesen, dass der Antisemitismus in der Revolution nach wie vor nur unzureichend erforscht ist. Allein Michael Brenner zeichnet am Beispiel von München diese Entwicklung minutiös nach. In Deutschland setzte eine massive Propagandawelle ein, in der dieser Antisemitismus, der im Krieg in deutschnationalen und deutsch-völkischen Zirkeln entwickelt worden war, in die politische Kultur und das öffentliche Leben eindrang und wahnhafte Züge annahm. Bezeichnenderweise war es die Zeit unmittelbar nach der Revolution, in der eine im zaristischen Russland kompilierte Legende, die „Protokolle der Weisen von Zion“, ihre breite europäische Rezeption erfuhr. Darüber hinaus wurde das Motiv des „Jüdischen Bolschewismus“ zu einem der wirkungsmächtigsten politischen Schlagworte des extremen Antisemitismus. Der Antisemitismus dieser Jahre beschränkte sich indes nicht auf diese völkischen und rassistischen Elemente, antisemitisches Denken war selbst im liberalen Bürgertum virulent. Dies macht nicht zuletzt der Brief von Ernst Troeltsch „Zur Judenfrage“ deutlich. ${ }^{76}$

So ist die Komplexität dieses Phänomens in den neueren Studien zur Revolution kaum erkannt. Vor allem sind die historischen Verbindungslinien zwischen dem völkischen Antisemitismus der frühen Nachkriegsjahre und der antisemitischen Politik der Nationalsozialisten nach der Machtübergabe noch immer unklar. Wenn Antisemitismus thematisiert wird, dann - trotz der Bekundungen, die Geschichte der Revolution nicht teleologisch von ihrem Ende her zu betrachten geschieht dies fast ausschließlich mit Blick auf das Dritte Reich. Erinnert sei an Robert Gerwarths Bemerkung, dass die negativen Urteile über die Revolution allein ,,vom retrospektiven Wissen um das Ende der Weimarer Republik vom Janu-

\footnotetext{
74 Jones: Anfang (wie Anm. 24), S. 253.

75 Krumeich: Niederlage (wie Anm. 12), S. 231.

76 Troeltsch: Vorherrschaft des Judentums?, in: ders.: Spectator-Briefe (wie Anm. 47), S. 209-217.
} 
ar 1933 geprägt" sind. ${ }^{77}$ Der konfliktreiche und gewalttätige Anfang der Republik musste nach Gerwarth nicht notwendigerweise auf 1933 zulaufen. Auch laut Michael Brenner führte von den Jahren 1918 bis 1923 ,kein direkter Weg ins Jahr 1933 “. ${ }^{78}$ Und doch erfuhren Juden im November 1923, so Brenner, den ,lebensbedrohlichen Schrecken des nationalsozialistischen Terrors. "79 Auch für Mark Jones brachte die Eskalation der Gewalt am Beginn der Republik ebenso nicht unmittelbar das nationalsozialistische Deutschland hervor. Dennoch schreibt Jones, dass die Gewaltphase „der Inkubationsraum für das Dritte Reich“ war. ${ }^{80}$

So bleibt es ein umso brennenderes Problem der historischen Forschung zu erklären, wie der im historischen Knoten von Krieg, Revolution und Konterrevolution entstandene extreme Antisemitismus nur ein Jahrzehnt später zum politischen Programm und zur Doktrin der Regierung des deutschen Staates werden konnte.

\section{Besprochene Literatur}

- Appel, Michael: Die letzte Nacht der Monarchie. Wie Revolution und Räterepublik in München Adolf Hitler hervorbrachten, 384 S., dtv, München 2018.

- Aulke, Julian: Räume der Revolution. Kulturelle Verräumlichung in Politisierungsprozessen während der Revolution 1918-1920 (Studien zur Geschichte des Alltags, Bd. 31), 483 S., Steiner, Stuttgart 2015.

- Benz, Wolfgang (Hrsg.): Die Novemberrevolution 1918/19. Intentionen - Strukturen - Wirkungen, in: Zeitschrift für Geschichtswissenschaft 66 (2018), H. 10, S. 797-858.

- Beutin, Heidi/Beutin, Wolfgang: Fanfaren einer neuen Freiheit. Deutsche Intellektuelle und die Novemberrevolution, 308 S., WBG, Darmstadt 2018.

- Bischoff, Frank/Hitze, Guido/Reininghaus, Wilfried (Hrsg).: Aufbruch in die Demokratie. Die Revolution 1918/19 im Rheinland und in Westfalen (Veröffentlichungen der Historischen Kommission für Westfalen, N.F., Bd. 51), 600 S., Aschendorff, Münster 2020.

- Braune, Andreas/Hesselbarth, Mario/Müller, Stefan (Hrsg.): Die USPD zwischen Sozialdemokratie und Kommunismus 1917-1922 (Weimarer Schriften zur Republik, Bd. 3), 262 S., Steiner, Stuttgart 2018.

- Braune, Andreas/Dreyer, Michael (Hrsg.): Zusammenbruch, Aufbruch, Abbruch? Die Novemberrevolution als Ereignis und Erinnerungsort (Weimarer Schriften zur Republik, Bd. 6), 326 S., Steiner, Stuttgart 2019.

- Brenner, Michael: Der lange Schatten der Revolution. Juden und Antisemiten in Hitlers München 1918 bis 1923, 400 S., Jüdischer Verlag, Berlin 2019.

\footnotetext{
77 Gerwarth: Revolutionen (wie Anm. 17), S. 15.

78 Brenner: Schatten (wie Anm. 40), S. 318.

79 Ebd., S. 282.

80 Jones: Anfang (wie Anm. 24), S. 343.
} 
- Derenthal, Ludger/Förster, Evelin/Kaufhold, Enno (Hrsg.): Berlin in der Revolution 1918/1919. Fotografie, Film, Unterhaltungskultur, 226 S., Kettler, Dortmund 2018.

- Dikovich, Albert/Wierzock, Alexander (Hrsg.): Von der Revolution zum Neuen Menschen. Das politische Imaginäre in Mitteleuropa 1918/19. Philosophie, Humanwissenschaften und Literatur (Weimarer Schriften zur Republik, Bd. 5), 347 S., Steiner, Stuttgart 2018.

- Dreyer, Michael/Braune, Andreas (Hrsg.): Weimar als Herausforderung. Die Weimarer Republik und die Demokratie im 21. Jahrhundert (Weimarer Schriften zur Republik, Bd. 1), 310 S., Steiner, Stuttgart 2016.

- Gerwarth, Robert: Die größte aller Revolutionen. November 1918 und der Aufbruch in eine neue Zeit, aus dem Engl. v. Alexander Weber, 384 S., Siedler, München 2018.

- Gietinger, Klaus: November 1918. Der verpasste Frühling des 20. Jahrhunderts, 272 S., Nautilus, Hamburg 2018.

- Görtz, Eva Bettina (Hrsg.): Eduard Bernsteins Briefwechsel mit Karl Kautsky (1912-1932), 633 S., Campus, Frankfurt a. M./New York 2011.

- Hirschfeld, Gerhard/Krumeich, Gerd/Renz, Irina (Hrsg.): 1918. Die Deutschen zwischen Weltkrieg und Revolution, 312 S., Links, Berlin 2018.

- Holzer, Anton (Hrsg.): Krieg nach dem Krieg. Revolution und Umbruch 1918/19, 192 S., Theiss, Darmstadt 2017.

- Jacob, Frank: Revolution und Räterepublik in Unterfranken. Eine landesgeschichtliche Untersuchung zu Verlauf und Folgen der Revolution von 1918/19 an der bayerischen Peripherie, 174 S., Königshausen \& Neumann, Würzburg 2019.

- Jones, Mark: Am Anfang war Gewalt. Die deutsche Revolution 1918/19 und der Beginn der Weimarer Republik, aus dem Engl. v. Karl Heinz Siber, 432 S., Propyläen, Berlin 2017 (engl. 2016).

- Käppner, Joachim: 1918. Aufstand für die Freiheit. Die Revolution der Besonnenen, 528 S., Piper, München 2017.

- Keil, Lars-Broder/Kellerhoff, Sven Felix: Lob der Revolution. Die Geburt der deutschen Demokratie, 288 S., Theiss/WBG, Darmstadt 2018.

- Kinzler, Sonja/Tillmann, Doris (Hrsg.): Die Stunde der Matrosen. Kiel und die deutsche Revolution 1918, 304 S., Theiss/WBG, Darmstadt 2018.

- Köhler, Thomas/Burmeister, Ralf/Nentwig, Janina (Hrsg.): Freiheit. Die Kunst der Novembergruppe 1918-1935, 272 S., Prestel, München 2018.

- Krumeich, Gerd: Die unbewältigte Niederlage. Das Trauma des Ersten Weltkriegs und die Weimarer Republik, 336 S., Herder, Freiburg i. Br. 2018.

- Kuckuk, Peter/Schröder, Ulrich: Bremen in der Deutschen Revolution 1918/1919. Revolution, Räterepublik, Restauration, überarb. und erw. Neuaufl., 508 S., Edition Falkenberg, Rotenburg/Wümme 22017 (orig. 1986).

- Langer, Bernd: Die Flamme der Revolution. Deutschland 1918/19, 444 S., Unrast, Münster 2018.

- Lehnert, Detlef (Hrsg.): Revolution 1918/19 in Norddeutschland (Historische Demokratieforschung, Bd. 13), 383 S., Metropol, Berlin 2018.

- Machtan, Lothar: Kaisersturz. Vom Scheitern im Herzen der Macht 1918, 352 S., Theiss/WBG, Darmstadt 2018. 
- Müller, Dirk H.: Die revolutionären Obleute und der November 1918. Zur Verschränkung von institutioneller Revolution und Rätebewegung, 350 S., BoD, Norderstedt 2019.

- Niess, Wolfgang: Die Revolution von 1918/19. Der wahre Beginn unserer Demokratie, 464 S., Europa, München/Wien 2017.

- Platthaus, Andreas: Der Krieg nach dem Krieg. Deutschland zwischen Revolution und Versailles 1918/19, 448 S., Rowohlt Berlin, Berlin 2018.

- Rackwitz, Martin: Kiel 1918. Revolution - Aufbruch zu Demokratie und Republik, 220 S., Wachholtz, Kiel 2018.

- Reininghaus, Wilfried: Die Revolution 1918/19 in Westfalen und Lippe als Forschungsproblem. Quellen und offene Fragen. Mit einer Dokumentation zu den Arbeiter-, Soldaten- und Bauernräten (Veröffentlichungen der Historischen Kommission für Westfalen, N. F., Bd. 33), 392 S., Aschendorff, Münster 2016.

- Rürup, Reinhard: Revolution und Demokratiegründung. Studien zur deutschen Geschichte 1918/19, mit einem Nachwort v. Peter Brandt/Detlef Lehnert, 247 S., Wallstein, Göttingen 2020.

- Schafmeister, Julia/Sunderbrink, Bärbel/Zelle, Michael (Hrsg.): Revolution in Lippe. 1918 und der Aufbruch in die Demokratie (Kataloge des Lippischen Landesmuseums Detmold, Bd. 23/Sonderveröffentlichungen des Naturwissenschaftlichen und Historischen Vereins für das Land Lippe, Bd. 94), 200 S., Verlag für Regionalgeschichte, Bielefeld 2018.

- Schöler, Uli/Scholle, Thilo (Hrsg.): Weltkrieg. Spaltung. Revolution. Sozialdemokratie 1916-1922, 472 S., Dietz Nachf., Bonn 2018.

- Schütrumpf, Jörn (Hrsg.): Diktatur statt Sozialismus. Die russische Revolution und die deutsche Linke 1917/18, 464 S., Dietz, Berlin 2017.

- Stalmann, Volker: Die Wiederentdeckung der Revolution von 1918/19. Forschungsstand und Forschungsperspektiven, in: Zeitschrift für Geschichtswissenschaft 64 (2016), H. 6, S. 521-541.

- Stamm-Kuhlmann, Thomas (Hrsg.): November 1918. Revolution an der Ostsee und im Reich (Veröffentlichungen der Historischen Kommission für Pommern. Reihe V: Forschungen zur Pommerschen Geschichte, Bd. 53), 285 S., Böhlau, Köln u. a. 2020.

- Troeltsch, Ernst: Kritische Gesamtausgabe, Bd. 14: Spectator-Briefe und Berliner Briefe (1919-1922), hrsg. v. Gangolf Hübinger, 739 S., De Gruyter, Berlin u.a. 2015.

- Weidermann, Volker: Träumer. Als die Dichter die Macht übernahmen, 288 S., Kiepenheuer \& Witsch, Köln 2017.

- Wirsching, Andreas/Kohler, Berthold/Wilhelm, Ulrich (Hrsg.): Weimarer Verhältnisse? Historische Lektionen für unsere Demokratie, 119 S., Reclam, Stuttgart 2018 .

Funding Open Access funding enabled and organized by Projekt DEAL.

Open Access Dieser Artikel wird unter der Creative Commons Namensnennung 4.0 International Lizenz veröffentlicht, welche die Nutzung, Vervielfältigung, Bearbeitung, Verbreitung und Wiedergabe in jeglichem Medium und Format erlaubt, sofern Sie den/die ursprünglichen Autor(en) und die Quelle ord- 
nungsgemäß nennen, einen Link zur Creative Commons Lizenz beifügen und angeben, ob Änderungen vorgenommen wurden.

Die in diesem Artikel enthaltenen Bilder und sonstiges Drittmaterial unterliegen ebenfalls der genannten Creative Commons Lizenz, sofern sich aus der Abbildungslegende nichts anderes ergibt. Sofern das betreffende Material nicht unter der genannten Creative Commons Lizenz steht und die betreffende Handlung nicht nach gesetzlichen Vorschriften erlaubt ist, ist für die oben aufgeführten Weiterverwendungen des Materials die Einwilligung des jeweiligen Rechteinhabers einzuholen.

Weitere Details zur Lizenz entnehmen Sie bitte der Lizenzinformation auf http://creativecommons.org/ licenses/by/4.0/deed.de.

Ulrich Wyrwa ist Professor für Neuere Geschichte an der Universität Potsdam. Von 2005 bis 2015 leitete er zusammen mit Werner Bergmann am Zentrum für Antisemitismusforschung der Technischen Universität Berlin zwei internationale Doktorandenkollegs zur Entstehung und Entwicklung des Antisemitismus in Europa (1879-1914/1914-1923). Er hatte Gastprofessuren am Fritz-Bauer-Institut der Goethe-Universität Frankfurt a. M., am Centrum für Jüdische Studien der Universität Graz und am Richard Koebner Minerva Center for German History an der Hebrew University Jerusalem inne. 